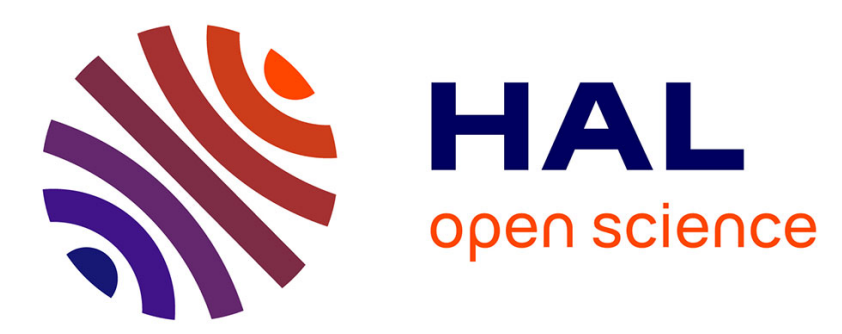

\title{
Scheduling and Buffer Sizing of n-Synchronous Systems
}

\author{
Louis Mandel, Florence Plateau
}

\section{To cite this version:}

Louis Mandel, Florence Plateau. Scheduling and Buffer Sizing of n-Synchronous Systems: Typing of Ultimately Periodic Clocks in Lucy-n. Eleventh International Conference on Mathematics of Program Construction (MPC'12), Jun 2012, Madrid, Spain. hal-01508142

\section{HAL Id: hal-01508142 \\ https://hal.inria.fr/hal-01508142}

Submitted on 13 Apr 2017

HAL is a multi-disciplinary open access archive for the deposit and dissemination of scientific research documents, whether they are published or not. The documents may come from teaching and research institutions in France or abroad, or from public or private research centers.
L'archive ouverte pluridisciplinaire HAL, est destinée au dépôt et à la diffusion de documents scientifiques de niveau recherche, publiés ou non, émanant des établissements d'enseignement et de recherche français ou étrangers, des laboratoires publics ou privés. 


\title{
Scheduling and Buffer Sizing of n-Synchronous Systems \\ Typing of Ultimately Periodic Clocks in Lucy-n
}

\author{
Louis Mandel Florence Plateau * \\ Laboratoire de Recherche en Informatique, Université Paris-Sud 11 \\ Laboratoire d'Informatique de l'École Normale Supérieure, INRIA
}

\begin{abstract}
Lucy-n is a language for programming networks of processes communicating through bounded buffers. A dedicated type system, termed a clock calculus, automatically computes static schedules of the processes and the sizes of the buffers between them.

In this article, we present a new algorithm which solves the subtyping constraints generated by the clock calculus. The advantage of this algorithm is that it finds schedules for tightly coupled systems. Moreover, it does not overestimate the buffer sizes needed and it provides a way to favor either system throughput or buffer size minimization.
\end{abstract}

\section{Introduction}

The n-synchronous model [8] is a data-flow programming model. It describes networks of processes that are executed concurrently and communicate through buffers of bounded size. It combines concurrency, determinism and flexible communications. These properties are especially useful for programming multimedia applications.

A language called Lucy-n [17] has been proposed for programming in the n-synchronous model. It is essentially Lustre [5] extended with a buffer operator. Lucy-n provides a static analysis that infers the activation conditions of computation nodes and the related sizes of buffers. This analysis is in the tradition of the clock calculus of the synchronous data-flow languages [10]. A clock calculus is a dedicated type system that ensures that a network of processes can be executed in bounded memory. The original clock calculus ensures that a network can be executed without buffering [6]. In the synchronous languages, each flow is associated with a clock that defines the instants where data is present. The clocks are infinite binary words where the occurrence of a 1 indicates the presence of a value on the flow and the occurrence of a 0 indicates the absence of a value. Here is an example of a flow $\mathrm{x}$ and its clock:

$$
\begin{array}{l|llllllll}
\mathrm{x} & 2 & 5 & 3 & 7 & 9 & 4 & 6 & 6
\end{array} \mid
$$

* Presently at Prove \& Run. 
The clock calculus forces each expression to satisfy a typing constraint similar to the following $\left(e_{1}+e_{2}\right.$ is the pointwise application of the addition operator +$)$ :

$$
\frac{H \vdash e_{1}: c t_{1}\left|C_{1} \quad H \vdash e_{2}: c t_{2}\right| C_{2}}{H \vdash e_{1}+e_{2}: c t_{3} \mid\left\{c t_{1}=c t_{2}=c t_{3}\right\} \cup C_{1} \cup C_{2}}
$$

This rule establishes that in the typing environment $H$, the expression $e_{1}+e_{2}$ has a clock of type $c t_{3}$ if $e_{1}$ has a clock of type $c t_{1}, e_{2}$ a clock of type $c t_{2}$ and if the constraint $c t_{1}=c t_{2}=c t_{3}$ is satisfied. ${ }^{1}$ Type equality ensures clock equality. Thus two processes producing flows of the same type can be composed without buffers.

The traditional clock calculus of synchronous languages only considers equality constraints on types; adapting the clock calculus to the n-synchronous model requires the introduction of a subtyping rule for the buffer primitive. If a flow whose clock is of type $c t$ can be stored in a buffer of bounded size to be consumed on a clock of type $c t^{\prime}$, we say that $c t$ is a subtype of $c t^{\prime}$, denoted $c t<: c t^{\prime}$ :

$$
\frac{H \vdash e: c t \mid C}{H \vdash \operatorname{buffer}(e): c t^{\prime} \mid\left\{c t<: c t^{\prime}\right\} \cup C}
$$

The clock calculus of Lucy-n considers both equality and subtyping constraints.

To solve such constraints, we have to be able to unify types $\left(c t_{1}=c t_{2}\right)$ and to verify the subtyping relation $\left(c t_{1}<: c t_{2}\right)$. These two operations depend very much on the clock language. One especially interesting and useful clock language can be built from ultimately periodic binary words which comprise a finite prefix followed by an infinite repetition of a finite pattern. An algorithm to solve constraints on the types of ultimately periodic clocks is proposed in [17]. The algorithm exploits clock abstraction [9] where the exact "shape" of clocks is forgotten in favor of simpler specifications of the presence instants of the flows: their asymptotic rate and two offsets bounding the potential delay with respect to this rate.

Type constraints on abstract clocks can be solved efficiently. But, the loss of precise information leads to over-approximations of buffer sizes. Moreover, even if a constraint system has a solution, the resolution algorithm can fail to find it because of the abstraction. Therefore, when clocks are simple, we prefer to find buffer sizes precisely, rather than quickly.

In this article, we present an algorithm to solve the constraints without clock abstraction. This problem is difficult for two reasons. First, such an algorithm must consider all the information present in the clocks. If the prefixes and periodic patterns of the words that describe the clocks are long, there may be combinatorial explosions. Second, the handling of the initial behaviors (described by the prefixes of the words) is always delicate [2] and not always addressed [1]. Dealing with the initial and periodic behaviors simultaneously is a source of complexity but, to the best of our knowledge, there is no approach that manages to treat them in separate phases.

$\overline{{ }^{1} \text { The sets } C_{1}}$ and $C_{2}$ contain the constraints collected during the typing of the expressions $e_{1}$ and $e_{2}$. 


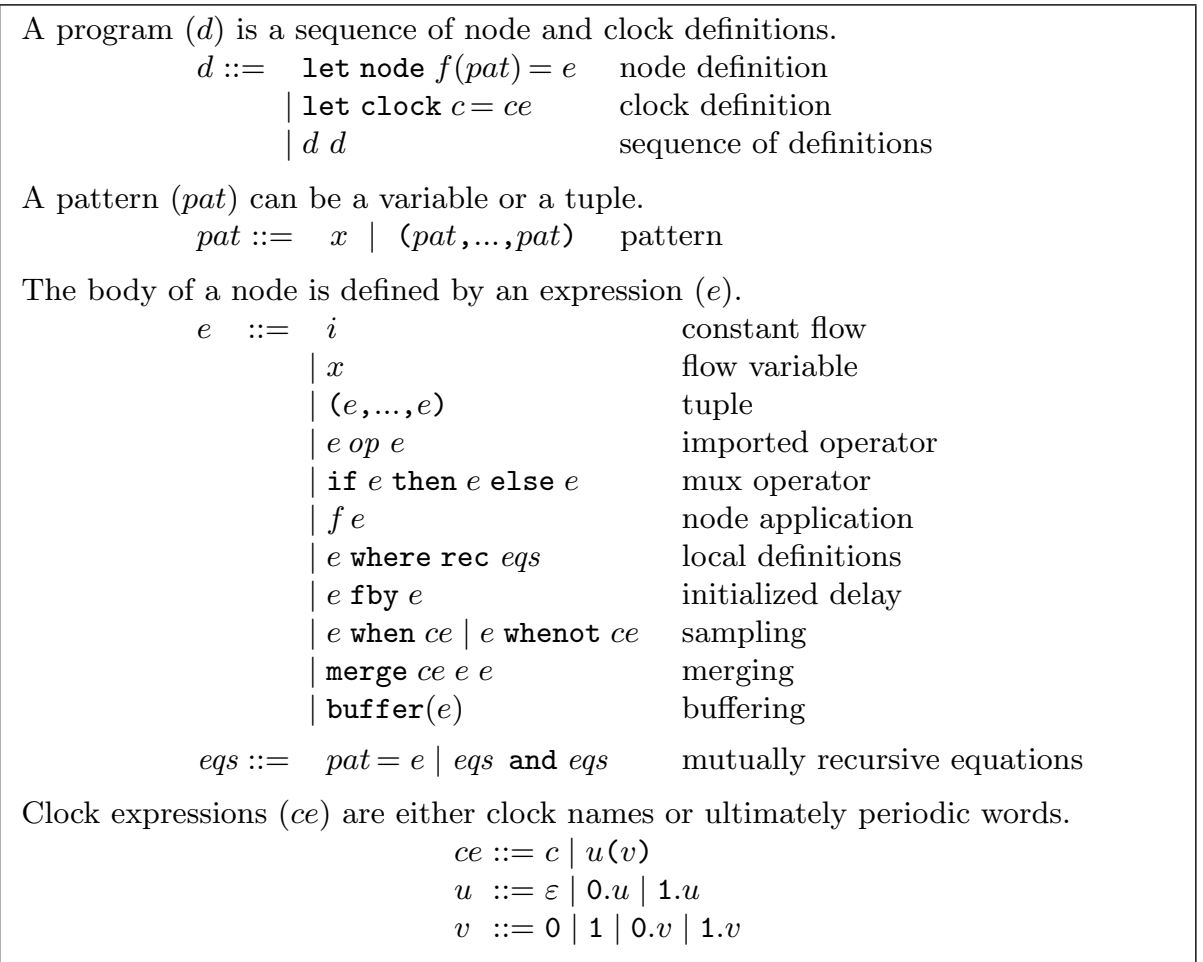

Fig. 1. The Lucy-n kernel.

Section 2 and 3 we present the Lucy-n language and its clock calculus by way of an extended example. Section 4 introduces the properties used in Section 5, which presents an algorithm for resolving constraints. Section 6 discusses results obtained on examples and compares them with previous resolution algorithms. Finally, Section 7 concludes the article.

An extended version of the article [16] with additional details and proofs, the code of the examples, a commented implementation of the algorithm and the Lucy-n compiler are available at http://www.lri.fr/ mandel/mpc12. ${ }^{2}$

\section{The Lucy-n Language}

The kernel of the Lucy-n language is summarized in Figure 1. In this section, we present the language through the programming of a GSM voice encoder component. This component is a cyclic encoder. It takes as input a flow of bits

\footnotetext{
${ }^{2}$ While the present paper is based on [15], it presents some new results. In particular, we generalize the first version of the algorithm, which allows us to define both a semi-decidable and complete algorithm and a decidable algorithm which is complete on a well defined class of systems. Finally, we also explain how to favor either system throughput or buffer size minimization.
} 


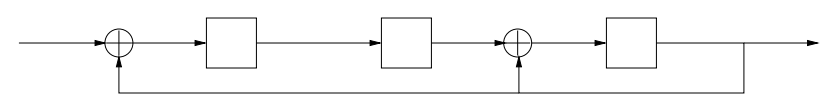

Fig. 2. Circuit for division [19] by $X^{3}+X+1$. The input flow is the sequence of fifty coefficients of the polynomial to divide. After consuming the fiftieth input bit, all the coefficients of the quotient polynomial have been produced at the output and the registers contain the coefficients of the remainder polynomial.

representing voice samples and produces an output flow that contains 3 new redundancy bits after every 50 data bits. The redundancy bits are the coefficients of the remainder of the division of the 50 bits to encode, considered as a polynomial of degree 49, by a polynomial peculiar to the encoder, here $X^{3}+X+1$.

The classical circuit to divide a polynomial is shown in Figure 2; the operator $\oplus$ represents the exclusive-or and boxes represent registers initialized to false.

The exclusive-or operator can be programmed as follows in Lucy-n (corresponding block diagrams are shown to the right of code samples):

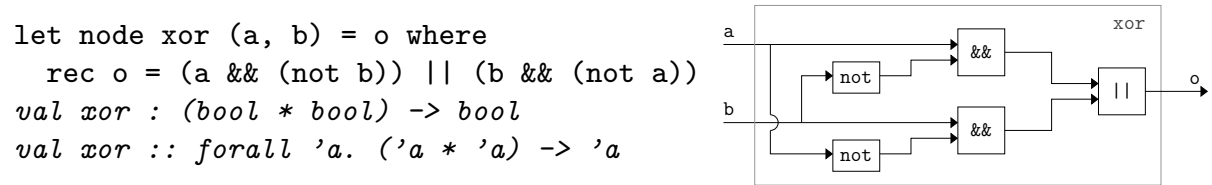

The node xor takes as input two flows $\mathrm{a}$ and $\mathrm{b}$ and computes the value of the output flow $\circ$. The value of $\mathrm{o}$ is defined by the equation $\mathrm{o}=(\mathrm{a} \& \&$ (not b) $)||$ (b \&\& (not a)) where the scalar operators \&\&, II and not are applied pointwise to their input flows. Hence, if we apply the node xor to two flows $\mathrm{x}$ and $\mathrm{y}$, we obtain a new flow $\operatorname{xor}(\mathrm{x}, \mathrm{y})$ :

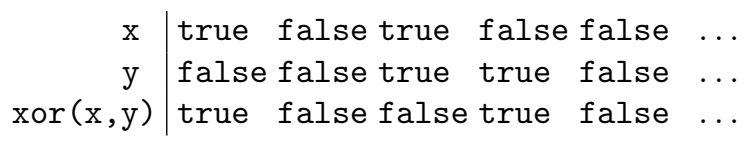

The definition of the xor node is followed by two facts automatically inferred by the Lucy-n compiler: the data type (val xor : (bool * bool) $\rightarrow$ bool), and the clock type (val xor : : forall 'a. ('a* 'a) $\rightarrow$ 'a). In the clock type, the variable ' $a$ represents the activation condition of the node. The type ' $a *$ ' $a$ $->$ ' $a$ means that at each activation, the two inputs are consumed (thus, they must be present) and the output is produced instantaneously. Since ' $a$ is a polymorphic variable, this type indicates that the node can be applied to any input flows that have the same clock as each other, whatever that clock is, and that it will have to be activated according to the instants defined by this clock.

Using this new node and the initialized register primitive of Lucy-n, fby (followed by), we can program the circuit of Figure 2. 


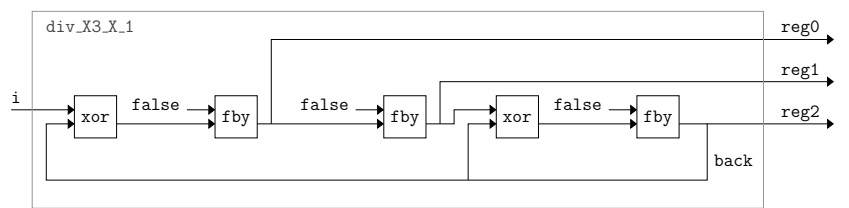

let node div_X3_X_1 $i=(\operatorname{reg} 0, r e g 1, r e g 2)$ where

rec $\operatorname{reg} 0=$ false fby $(\operatorname{xor}(i$, back $))$

and reg $1=$ false $f$ by $r e g 0$

and $r e g 2=$ false fby $(\operatorname{xor}(r e g 1$, back $))$

and back $=$ reg 2

val div_X3_X_1 : bool $\rightarrow$ (bool * bool * bool)

val div_X3_X_1 : forall ' $a$. ' $a \rightarrow$ ('a* 'a* 'a)

The equation reg1 $=$ false $\mathrm{fby}$ reg 0 means that reg 1 is equal to false at the first instant and equal to the preceding value of reg0 at the following instants. Note that the definitions of flows reg0, reg1, reg2 and back are mutually recursive.

In order to divide a flow of polynomials, the div_X3_X_1 node must be modified. After the arrival of the coefficients of each polynomial, that is after every 50 input bits, the three registers must be reset to false. Since the content of some registers is the result of an exclusive-or between the feedback edge back and the preceding register (or the input flow for the first register), to reset the registers to false, we have to introduce three false values as input and three false values on the feedback wire, every 50 input bits. ${ }^{3}$

The clock type of the node div_X3_X_1 modified accordingly is: ${ }^{4}$ val div_X3_X_1 : forall ' $a$. ' $a$ on (1^50 0^3) $\rightarrow$ ('a * 'a* 'a) The notation (1^50 $0^{-3}$ ) represents the infinite repetition of the binary word $1^{50} 0^{3}$ where $1^{50}$ is the concatenation of fifty 1 s and $0^{3}$ the concatenation of three 0s. To understand the type of div_X3_X_1, notice that ' $a$ (the activation rhythm of the node) defines the notion of instants for the equations of the node. The clock type of the input flow is ' $a$ on (1 $1^{-5}$ 0 0 3). It means that the input flow has to be present during the first 50 instants, then absent for 3 instants (during which the registers are reset). Therefore, this node can compute one division every 53 instants of the rhythm ' $a$. Finally, the clock type of the three outputs is ' $a$, it means that the values of the registers are produced at each instant.

Now, to define a node redundancy which computes only the redundancy bits corresponding to a flow of polynomials, we sample the output of the node div_X3_X_1. In our implementation of the node div_X3_X_1, the remainder of the division is contained in the registers after the 50th input bit and output at the 51st instant. Thus, the redundancy node has to sample the output of div_X3_X_1 at the 51st instant. For this, we use the when operator. It is parameterized by a flow and a sampling condition, and it filters the values of the flow following the pattern defined by the sampler: if the flow is absent, the output of the when is absent; if the input flow is present and the next element of the sampler is 1 , the

\footnotetext{
${ }^{3}$ It is implicit, here and in the following, that such behaviors iterate repeatedly.

4 The source code of the modified node is available at http://www.lri.fr/ mandel/ mpc12/gsm. ls.html.
} 
value of the flow is output; if the input flow is present and the next element of the sampler is 0 , the output of the when is absent. To keep only the 51st element of a sequence of $53 \mathrm{bits}$, we use the sampling condition $\left(0^{50} 100\right)$ :

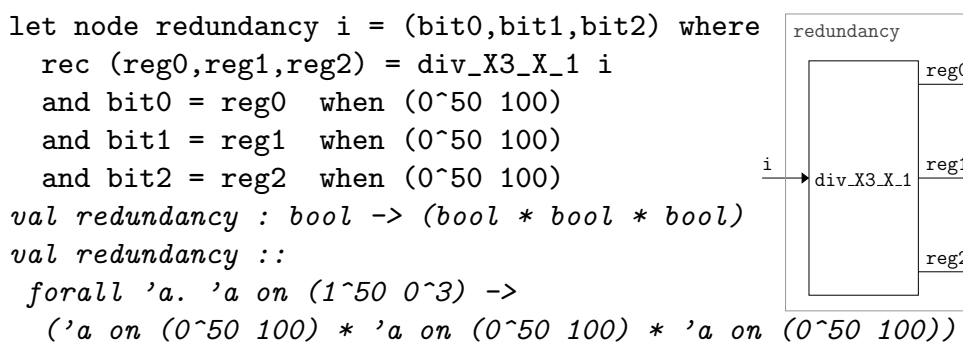

To append 3 redundancy bits after 50 data bits, we use the merge operator. Its parameters are a merging condition and two flows; merge ce $e_{1} e_{2}$ outputs the value of $e_{1}$ when $c e$ is equal to 1 and the value of $e_{2}$ when ce is equal to 0 . The flows $e_{1}$ and $e_{2}$ must be present on disjoint instants of the clock of $c e$ : when $c e$ is equal to $1, e_{1}$ must be present and $e_{2}$ absent and vice versa when ce is equal to 0 . Thus, to incorporate the first redundancy bit (bit0) after 50 input bits, we use the merging condition $\left(1^{50} 0\right)$ and obtain a flow of 51 bits. Then, we use the condition $\left(1^{51} 0\right)$ to incorporate the second redundancy bit, and finally the condition $\left(1^{52} 0\right)$ for the third redundancy bit.

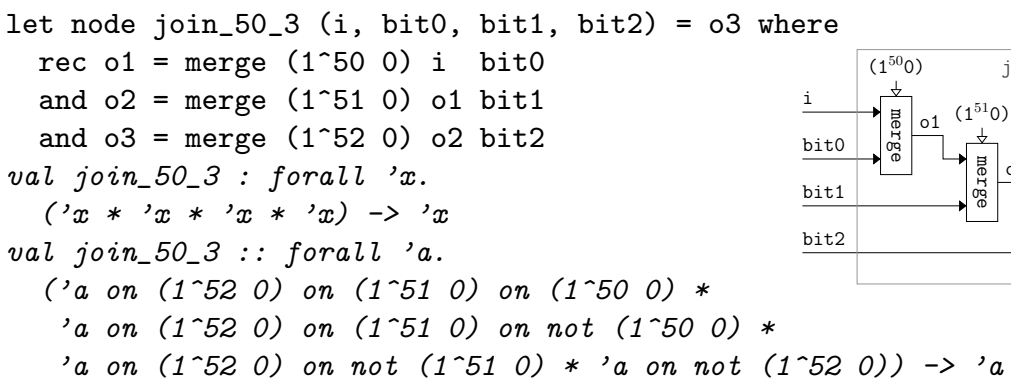

We will see in Section 4 that the clock type of join_50_3 is equivalent to: $\forall \alpha .\left(\alpha\right.$ on $\left(1^{50} 000\right) \times \alpha$ on $\left(0^{50} 100\right) \times \alpha$ on $\left(0^{50} 010\right) \times \alpha$ on $\left.\left(0^{50} 001\right)\right) \rightarrow \alpha$ This type expresses that the flow containing data must be present for the first 50 instants, and then absent for the following 3 instants. The flows containing the first, second and third redundancy bits must arrive at the 51st, 52nd, and 53rd instants respectively.

To complete the cyclic encoder, we must use the node redundancy to compute the three redundancy bits and the node join_50_3 to incorporate them into the input flow. But the redundancy bits are produced at instant 51 which is too early for the join_50_3 node which expects them successively at instants 51, 52 and 53. They must thus be stored using the buffer operator: 


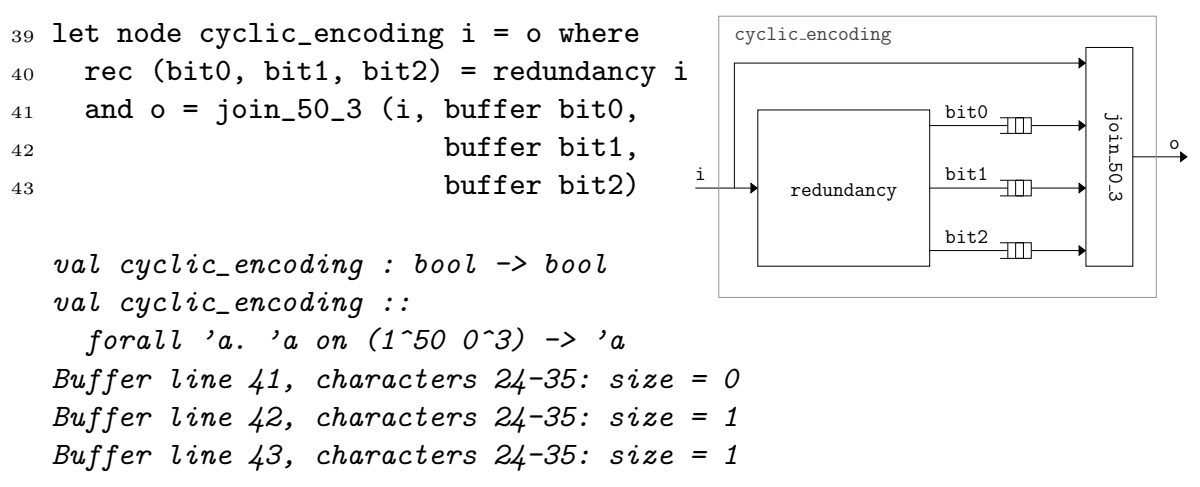

The compiler automatically computes the buffer sizes required. We can see that the buffer at line 41 is not really needed, the inferred size is 0 . This buffer is used for the communication of the first redundancy bit (bit0) between the redundancy node and the join_50_3 node. This bit is produced at the 51st instant and consumed immediately. The two other redundancy bits (bit1 and bit2) are also produced at the 51st instant, but they are consumed later. Thus the second bit has to be stored in a buffer of size 1 for 1 instant and the third bit has to be stored in a buffer of size 1 for 2 instants.

Notice that before calculating the buffer sizes, the compiler must infer the activation rhythm of each node. When the output of one node is consumed directly by another, i.e., when there is no buffer between them, the nodes must be activated such that the outputs of the first node are produced at the very same instants that they are to be consumed as inputs by the second node. When the output of one node is consumed by another through a buffer, the nodes must be activated such that the buffer is not read when it is empty and such that there is no infinite accumulation of data in the buffer.

\section{Clock Calculus}

We have seen in Section 1 that each expression in a program must satisfy a type constraint (the rules of the clock calculus are detailed in annex A). To illustrate the typing inference algorithm which collects the constraints, we return to the cyclic_encoding node of the previous section.

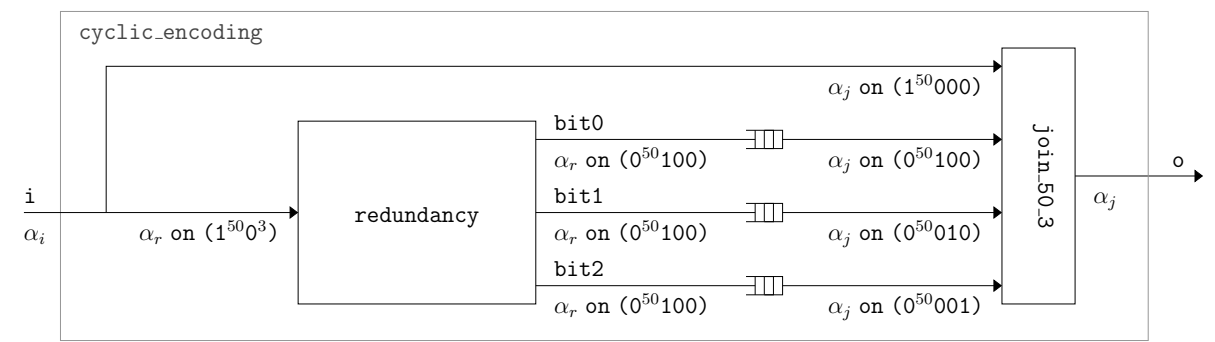

If we associate with the input $i$ the clock type variable $\alpha_{i}$, the expression redundancy $i$ generates the equality constraint $\alpha_{i}=\alpha_{r}$ on $\left(1^{50} 0^{3}\right)$. Indeed, 
once instantiated with a fresh variable $\alpha_{r}$, the clock type of the node redundancy is $\alpha_{r}$ on $\left(1^{50} 0^{3}\right) \rightarrow\left(\alpha_{r}\right.$ on $\left(0^{50} 100\right) \times \alpha_{r}$ on $\left(0^{50} 100\right) \times \alpha_{r}$ on $\left.\left(0^{50} 100\right)\right)$. Hence, the type of its input must be equal to $\alpha_{r}$ on $\left(1^{50} 0^{3}\right)$. Consequently, the equation (bit0, bit1, bit2) = redundancy $i$ adds to the typing environment that bit0, bit1 and bit2 are of type $\alpha_{r}$ on $\left(0^{50} 100\right)$.

Similarly, the application of join_50_3 adds some constraints on the types of its inputs. Once instantiated with a fresh type variable $\alpha_{j}$, the clock type of the node join_50_3 is $\left(\alpha_{j}\right.$ on $\left(1^{50} 000\right) \times \alpha_{j}$ on $\left(0^{50} 100\right) \times \alpha_{j}$ on $\left(0^{50} 010\right) \times$ $\alpha_{j}$ on $\left.\left(0^{50} 001\right)\right) \rightarrow \alpha_{j}$. This type imposes the constraint that the type of the first input (here $\alpha_{i}$, the type of the data input i) has to be equal to $\alpha_{j}$ on $\left(1^{50} 000\right)$ and the types of the other inputs (here $\alpha_{r}$ on $\left.\left(0^{50} 100\right)\right)$ must be, respectively, subtypes of $\alpha_{j}$ on $\left(0^{50} 100\right), \alpha_{j}$ on $\left(0^{50} 010\right)$ and $\alpha_{j}$ on $\left(0^{50} 001\right)$. For these last inputs, we do not impose type equality but rather only subtyping $(<:)$ since they are consumed through buffers. The subtyping relation ensures that there are neither reads in an empty buffer nor writes in a full buffer. Finally, the equation $0=$ join_50_3 (..) augments the typing environment with the information that the type of $\circ$ is $\alpha_{j}$, the return type of join_50_3.

The cyclic_encoding node thus has the clock type $\alpha_{i} \rightarrow \alpha_{j}$, with the following constraints:

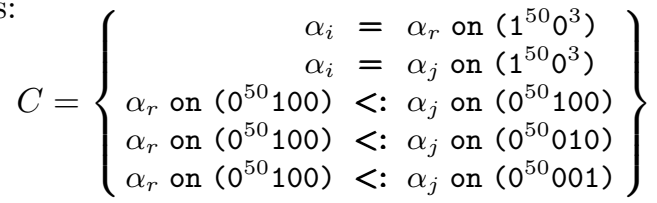

To finish the typing of this node and to be able to compute the buffer sizes, we have to find a solution to this constraint system, that is we must find instantiations of the variables $\alpha_{i}, \alpha_{r}$ and $\alpha_{j}$ such that the constraints are always satisfied. These instantiations have to be Lucy-n clock types, i.e., of the shape: ct ::= $\alpha \mid($ ct on $p)$ where $p$ is an ultimately periodic binary word (formally defined in Section 4.1).

To solve the constraint system of the example, we start with the equality constraints and choose the following substitution: $\theta=\left\{\alpha_{i} \leftarrow \alpha\right.$ on $\left(1^{50} 0^{3}\right) ; \alpha_{r} \leftarrow \alpha$; $\left.\alpha_{j} \leftarrow \alpha\right\}$. Applying this substitution to $C$ gives:

$$
\theta(C)=\left\{\begin{array}{c}
\alpha \text { on }\left(1^{50} 0^{3}\right)=\alpha \text { on }\left(1^{50} 0^{3}\right) \\
\alpha \text { on }\left(1^{50} 0^{3}\right)=\alpha \text { on }\left(1^{50} 0^{3}\right) \\
\alpha \text { on }\left(0^{50} 100\right)<: \alpha \text { on }\left(0^{50} 100\right) \\
\alpha \text { on }\left(0^{50} 100\right)<: \alpha \text { on }\left(0^{50} 010\right) \\
\alpha \text { on }\left(0^{50} 100\right)<: \alpha \text { on }\left(0^{50} 001\right)
\end{array}\right\} \Leftrightarrow\left\{\begin{array}{l}
\alpha \text { on }\left(0^{50} 100\right)<: \alpha \text { on }\left(0^{50} 100\right) \\
\alpha \text { on }\left(0^{50} 100\right)<: \alpha \text { on }\left(0^{50} 010\right) \\
\alpha \text { on }\left(0^{50} 100\right)<: \alpha \text { on }\left(0^{50} 001\right)
\end{array}\right\}
$$

Remark 1. Notice that there is no complete greedy unification algorithm because there is no most general unifier for clock types [21]. Therefore, to be complete, a resolution algorithm must take into account all the constraints globally. As in this example greedy structural unification leads to a solution, we used it for the sake of conciseness. In the general case, a simple way to handle equality constraints is to consider them as two subtyping constraints $\left(c t_{1}=c t_{2} \Leftrightarrow\right.$ $\left.\left(c t_{1}<: c t_{2}\right) \wedge\left(c t_{2}<: c t_{1}\right)\right)$. 


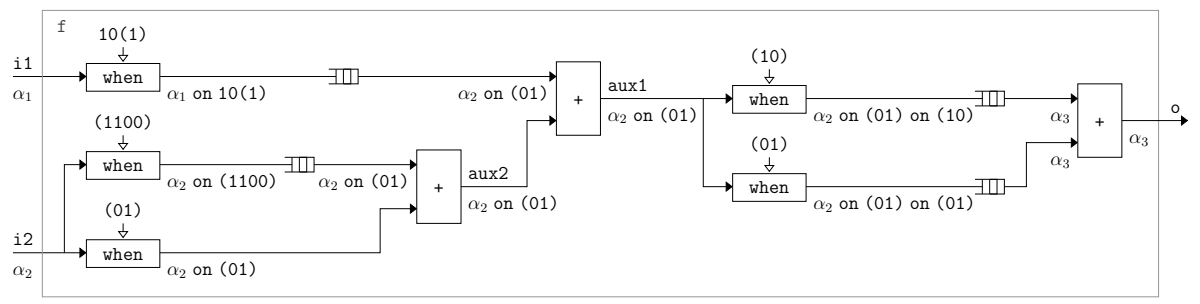

let node $f(i 1, i 2)=0$ where

rec aux1 = buffer (i1 when 10(1)) + aux2

and aux $2=$ buffer (i2 when (1100)) + i2 when (01)

and $0=$ buffer $(\operatorname{aux} 1$ when $(10))+$ buffer (aux1 when (01))

Fig. 3. The node $f$ and its block diagram representation. The diagram is annotated with the types obtained after the resolution of equality constraints.

After transforming our constraint system to a system that contains only subtyping constraints, we notice that all the constraints depend on the same type variable. So, we apply a result from [17] to simplify the on operators:

$$
\theta(C) \Leftrightarrow\left\{\begin{array}{l}
\left(0^{50} 100\right)<:\left(0^{50} 100\right) \\
\left(0^{50} 100\right)<:\left(0^{50} 010\right) \\
\left(0^{50} 100\right)<:\left(0^{50} 001\right)
\end{array}\right\}
$$

We will see in Section 5.1 that these constraints on words can be checked. Sometimes however, subtyping constraints are not expressed with respect to the same type variable. For example, the program of Figure 3 generates the following set of subtyping constraints where only the second constraint can be simplified:

$C^{\prime}=\left\{\begin{aligned} \alpha_{1} \text { on } 10(1) & <: \alpha_{2} \text { on (01) } \\ \alpha_{2} \text { on }(1100) & <: \alpha_{2} \text { on (01) } \\ \alpha_{2} \text { on }(01) \text { on (10) } & <: \alpha_{3} \text { on (1) } \\ \alpha_{2} \text { on (01) on (01) } & <: \alpha_{3} \text { on (1) }\end{aligned}\right\} \Leftrightarrow\left\{\begin{aligned} \alpha_{1} \text { on } 10(1) & <: \alpha_{2} \text { on (01) } \\ (1100) & <:(01) \\ \alpha_{2} \text { on (01) on (10) } & <: \alpha_{3} \text { on (1) } \\ \alpha_{2} \text { on (01) on (01) } & <: \alpha_{3} \text { on (1) }\end{aligned}\right\}$

But, in fact, such systems can always be reduced to ones where all the constraints are expressed with respect to a single type variable. To do so, we introduce word variables denoted $c_{n}$ and we replace each type variable $\alpha_{n}$ with $\alpha$ on $c_{n}$. Here, the application of the substitution $\theta=\left\{\alpha_{1} \leftarrow \alpha\right.$ on $c_{1} ; \alpha_{2} \leftarrow \alpha$ on $c_{2} ; \alpha_{3} \leftarrow \alpha$ on $\left.c_{3}\right\}$ to system $C^{\prime}$ gives:

$$
\begin{aligned}
& \theta\left(C^{\prime}\right)=\left\{\begin{aligned}
\alpha \text { on } c_{1} \text { on } 10(1) & <: \alpha \text { on } c_{2} \text { on (01) } \\
(1100) & <: \text { (01) } \\
\alpha \text { on } c_{2} \text { on (01) on }(10) & <: \alpha \text { on } c_{3} \text { on (1) } \\
\alpha \text { on } c_{2} \text { on (01) on (01) } & <: \alpha \text { on } c_{3} \text { on (1) }
\end{aligned}\right\} \\
& \Leftrightarrow\left\{\begin{aligned}
c_{1} \text { on } 10(1) & <: c_{2} \text { on }(01) \\
(1100) & <:(01) \\
c_{2} \text { on }(01) \text { on }(10) & <: c_{3} \text { on }(1) \\
c_{2} \text { on }(01) \text { on }(01) & <: c_{3} \text { on }(1)
\end{aligned}\right\}
\end{aligned}
$$

This succession of operations transforms a system where the unknowns are types into a system where the unknowns are ultimately periodic binary words. The operator on and the relation <: on binary words are defined in the following section. The algorithm that infers ultimately periodic binary words $c_{n}$ to satisfy the $<$ : relation is presented in Section 5 . 


\section{Algebra of Ultimately Periodic Words}

In this section, we present the definitions and properties of ultimately periodic binary words that underlie the constraint resolution algorithm presented in Section 5. Proofs are provided in the extended version of the article [16].

\subsection{Ultimately Periodic Binary Words}

We write $w$ for an infinite binary word $(w::=0 w \mid 1 w)$, $u$ or $v$ for finite binary words $(u, v::=0 u|1 u| \varepsilon),|u|$ for the size of $u$ and $|u|_{1}$ for the number of 1 s it contains. The buffer analysis relies on the instants of presence of data on the flows. Therefore, it mainly manipulates indexes of $1 \mathrm{~s}$ in the words:

Definition 1 (index of the $j$ th 1 in $w: \mathcal{I}_{w}(j)$ ).

Let $w$ be a binary word that contains infinitely many $1 s$.

$$
\begin{aligned}
& \mathcal{I}_{w}(0) \stackrel{\text { def }}{=} 0 \\
& \mathcal{I}_{w}(1) \stackrel{\text { def }}{=} 1 \quad \text { if } w=1 w^{\prime} \\
& \forall j>1, \mathcal{I}_{w}(j) \stackrel{\text { def }}{=} 1+\mathcal{I}_{w^{\prime}}(j-1) \text { if } w=1 w^{\prime} \\
& \forall j>0, \mathcal{I}_{w}(j) \stackrel{\text { def }}{=} 1+\mathcal{I}_{w^{\prime}}(j) \quad \text { if } w=0 w^{\prime}
\end{aligned}
$$

For example, the index of the third 1 in $w_{1}=1101011010 \ldots$ is 4 , i.e., $\mathcal{I}_{w_{1}}(3)=4$.

Remark 2 (increasing indexes). $\mathcal{I}_{w}$ is increasing: $\forall j \geq 1, \mathcal{I}_{w}(j)<\mathcal{I}_{w}(j+1)$.

Remark 3 (sufficient indexes). As a direct consequence of Remark 2, the index of the $j$ th 1 is greater than or equal to $j: \forall j \geq 1, \mathcal{I}_{w}(j) \geq j$.

A word $w$ can also be characterized by its cumulative function which counts the number of 1 s since the beginning of $w$.

Definition 2 (cumulative function of $\left.w: \mathcal{O}_{w}\right){ }^{5}$

$$
\mathcal{O}_{w}(0) \stackrel{\text { def }}{=} 0 \quad \forall i \geq 1, \mathcal{O}_{w}(i) \stackrel{\text { def }}{=} \sum_{0 \leq i^{\prime} \leq i} w\left[i^{\prime}\right]
$$

In this article, we consider ultimately periodic clocks $u(v)$ which comprise a finite word $u$ as prefix followed by the infinite repetition of a non-empty finite word $v$ :

Definition 3 (ultimately periodic word). $p=u(v) \stackrel{\text { def }}{\Leftrightarrow} p=u w$ with $w=v w$ For example, $p=1101(110)=1101110110110 \ldots$ We use the notation $p . u$ for the prefix of a word $p$ (e.g. $(1101(110)) . u=1101)$ and $p . v$ for its periodic pattern (e.g. $(1101(110)) \cdot v=110)$.

An ultimately periodic binary word has an infinite number of different representations. For example, $(10)=(1010)=1(01)=\ldots$. But, there exists a normal form which is the representation where the prefix and the periodic

\footnotetext{
${ }^{5}$ The notation $w[i]$ represents the $i$ th element of a word $w$.
} 
pattern have the shortest size. For some binary operations, however, it is more convenient to put the two words in a form which is longer than the normal form. For example, for some operations we would prefer that the operands have the same size, or the same number of $1 \mathrm{~s}$, or even that the number of $1 \mathrm{~s}$ in the first word is equal to the size of the second word.

Remark 4. We can change the shape of an ultimately periodic binary word with the following manipulations:

- Increase prefix size: $u\left(v v^{\prime}\right)=u v\left(v^{\prime} v\right)$. For example, we can add two elements to the prefix of the word $p=1101$ (110) to obtain the form $110111(011)$. Increasing the size of the prefix can be used to increase the number of $1 \mathrm{~s}$ it contains.

- Repeat periodic pattern: $u(v)=u\left(v^{k}\right)$ with $k>0$. For example, we can triple the size of the periodic pattern of $p=1101$ (110) (and thus triple its number of 1s) to obtain 1101(110 110110$)$.

The following two properties ensure that a periodic word is well formed.

Remark 5 (periodicity). Two successive occurrences of the same 1 of a periodic pattern are separated by a distance equal to the size of the pattern:

$$
\forall j>|p . u|_{1}, \mathcal{I}_{p}\left(j+|p . v|_{1}\right)=\mathcal{I}_{p}(j)+|p . v|
$$

As a direct consequence of this property, the distance between any 1 in a repetition of a periodic pattern and the corresponding 1 in the first occurrence of the pattern is a multiple of the size of the pattern.

$$
\forall j,|p . u|_{1}<j \leq|p . u|_{1}+|p . v|_{1}, \mathcal{I}_{p}\left(j+l \times|p . v|_{1}\right)=\mathcal{I}_{p}(j)+l \times|p . v|
$$

For example, if $p=101(10010), \mathcal{I}_{p}(3+2)=\mathcal{I}_{p}(3)+5$ and $\mathcal{I}_{p}(4+2 \times 2)=$ $\mathcal{I}_{p}(3)+2 \times 5$.

Remark 6 (sufficient size). The size of the periodic pattern of a word $p$ (i.e., $|p . v|$ ) is greater than or equal to the number of elements between the indexes of the first and last 1 of the periodic pattern of $p$ (for words with at least one 1 in the periodic pattern):

$$
|p . v| \geq 1+\mathcal{I}_{p}\left(|p . u|_{1}+|p . v|_{1}\right)-\mathcal{I}_{p}\left(|p . u|_{1}+1\right)
$$

For example, if $p=101$ (10010), $|p \cdot v| \geq 1+7-4$.

The rate of a word $w$ is the proportion of $1 \mathrm{~s}$ in the word $w$ :

Definition 4 (rate of $p$ ). $\operatorname{rate}(w)=\lim _{i \rightarrow+\infty} \frac{\mathcal{O}_{w}(i)}{i}$

For an ultimately periodic binary word $p$, the rate is the ratio between the number of $1 \mathrm{~s}$ and the size of its periodic pattern:

Proposition 1 (rate of $p$ ). $\operatorname{rate}(p)=\frac{|p \cdot v|_{1}}{|p \cdot v|}$

In the following, we only consider words of non-null rate, i.e., such that the periodic pattern contains at least one 1 (these words have an infinite number of $1 \mathrm{~s}$ and are the clocks of flows that produce values infinitely often). 


\subsection{Adaptability Relation}

We now define the relation $<$ : on binary words, called the adaptability relation. The relation $w_{1}<: w_{2}$ holds if and only if a flow of clock $w_{1}$ can be stored in a buffer of bounded size and consumed at the rhythm of the clock $w_{2}$. It means that data does not accumulate without finite bound in the buffer, and that reads are not attempted when the buffer is empty. The adaptability relation is the conjunction of precedence and synchronizability relations. The synchronizability relation between two words $w_{1}$ and $w_{2}$ (written $w_{1} \bowtie w_{2}$ ) asserts that there is a finite upper bound on the number of values present in the buffer during an execution. It states that the asymptotic numbers of reads and writes from and to the buffer are equal. The precedence relation between the words $w_{1}$ and $w_{2}$ (written $w_{1} \preceq w_{2}$ ) asserts the absence of reads from an empty buffer. It states that the $j$ th write to the buffer always occurs before the $j$ th read.

Two words $w_{1}$ and $w_{2}$ are synchronizable if the difference between the number of occurrences of $1 \mathrm{~s}$ in $w_{1}$ and the number of occurrences of $1 \mathrm{~s}$ in $w_{2}$ is bounded.

\section{Definition 5 (synchronizability $\bowtie$ ).}

$$
w_{1} \bowtie w_{2} \stackrel{\text { def }}{\Leftrightarrow} \exists b_{1}, b_{2}, \forall i \geq 0, b_{1} \leq \mathcal{O}_{w_{1}}(i)-\mathcal{O}_{w_{2}}(i) \leq b_{2}
$$

To test this synchronizability relation on ultimately periodic binary words, we have only to check that the periodic patterns of the two words have the same proportion of 1s. For example, 1(1100) $\bowtie(101001)$ because $\frac{2}{4}=\frac{3}{6}$.

Proposition 2 (synchronizability test). $p_{1} \bowtie p_{2} \Leftrightarrow \operatorname{rate}\left(p_{1}\right)=\operatorname{rate}\left(p_{2}\right)$

A word $w_{1}$ precedes a word $w_{2}$ if the $j$ th 1 of $w_{1}$ always occurs before or at the same time as the $j$ th 1 of $w_{2}$.

Definition 6 (precedence $\preceq$ ). $w_{1} \preceq w_{2} \stackrel{\text { def }}{\Leftrightarrow} \forall j \geq 1, \mathcal{I}_{w_{1}}(j) \leq \mathcal{I}_{w_{2}}(j)$

To check this relation on ultimately periodic words, we only have to consider this relation until a "common" periodic behavior is reached. ${ }^{6}$ For example, $1(1100) \preceq(110100)$ because $\mathcal{I}_{1(1100)}(j) \leq \mathcal{I}_{(110100)}(j)$ for all $j$ such that $1 \leq j \leq 7$ and the relative behavior between the two words from the 8th 1 is exactly the same as the one from the 2 nd 1 . It can be seen if we rewrite $1(1100)$ as $1(110011001100)$ and $(110100)$ as $1(101001101001)$, the periodic patterns within these two words recommence simultaneously.

Proposition 3 (precedence test). Consider $p_{1}$ and $p_{2}$ such that $p_{1} \bowtie p_{2}$. Let $h=\max \left(\left|p_{1} \cdot u\right|_{1},\left|p_{2} \cdot u\right|_{1}\right)+\operatorname{lcm}\left(\left|p_{1} \cdot v\right|_{1},\left|p_{2} \cdot v\right|_{1}\right)$. Then:

$$
p_{1} \preceq p_{2} \quad \Leftrightarrow \quad \forall j, 1 \leq j \leq h, \mathcal{I}_{p_{1}}(j) \leq \mathcal{I}_{p_{2}}(j)
$$

The intuition for the value of the bound $h$ is the following. By Remark 4 , we can adjust the respective components of $p_{1}$ and $p_{2}$ to have the same number of $1 \mathrm{~s}$. We obtain two words $p_{1}^{\prime}$ and $p_{2}^{\prime}$ (equivalent to $p_{1}$ and $p_{2}$ ) such that the

\footnotetext{
${ }^{6}$ A common periodic behavior of two ultimately periodic words $p_{1}$ and $p_{2}$ is defined by an index $h$ and a size $k$ such that $\forall j>h, \mathcal{I}_{p_{2}}(j)-\mathcal{I}_{p_{1}}(j)=\mathcal{I}_{p_{2}}(j-k)-\mathcal{I}_{p_{1}}(j-k)$.
} 
number of $1 \mathrm{~s}$ in their prefixes is $\max \left(\left|p_{1} . u\right|_{1},\left|p_{2} . u\right|_{1}\right)$ and the number of $1 \mathrm{~s}$ in their periodic pattern is $\operatorname{lcm}\left(\left|p_{1} \cdot v\right|_{1},\left|p_{2} . v\right|_{1}\right)$. Hence, after the traversal of $h 1 \mathrm{~s}$ in $p_{1}^{\prime}$ and $p_{2}^{\prime}$ (with $h=\left|p_{1}^{\prime} \cdot u\right|_{1}+\left|p_{1}^{\prime} \cdot v\right|_{1}=\left|p_{2}^{\prime} \cdot u\right|_{1}+\left|p_{2}^{\prime} \cdot v\right|_{1}$ ), the periodic patterns of both words restart simultaneously. And since the two words have the same rate, we are in exactly the same situation as we were at the beginning of the first traversal of the periodic patterns. So, if the condition holds until the $h$ th 1 , it always holds.

The adaptability relation is the conjunction of the synchronizability and precedence relations.

Definition 7 (adaptability test). $p_{1}<: p_{2} \Leftrightarrow p_{1} \bowtie p_{2} \wedge p_{1} \preceq p_{2}$

\subsection{Buffer Size}

To compute the size of a buffer, we must know the number of values that are written and read during an execution.

Consider a buffer that takes as input a flow with clock $w_{1}$, and gives as output the same flow but with clock $w_{2}$. The number of elements present at each instant $i$ in the buffer is the difference between the number of values that have been written into it $\left(\mathcal{O}_{w_{1}}(i)\right)$ and the number of values that have been read from it $\left(\mathcal{O}_{w_{2}}(i)\right)$. The necessary and sufficient buffer size is the maximum number of values present in the buffer during any execution.

Definition 8 (buffer size). $\operatorname{size}\left(w_{1}, w_{2}\right)=\max _{i \in \mathbb{N}}\left(\mathcal{O}_{w_{1}}(i)-\mathcal{O}_{w_{2}}(i)\right)$

To compute this size on adaptable ultimately periodic binary words, we need only to consider the initial patterns of the two words before their "common" periodic behavior is reached.

Proposition 4 (buffer size).

Consider $p_{1}$ and $p_{2}$ such that $p_{1}<: p_{2}$.

Let $H=\max \left(\left|p_{1} \cdot u\right|,\left|p_{2} \cdot u\right|\right)+\operatorname{lcm}\left(\left|p_{1} \cdot v\right|,\left|p_{2} \cdot v\right|\right)$. Then:

$$
\operatorname{size}\left(p_{1}, p_{2}\right)=\max _{1 \leq i \leq H}\left(\mathcal{O}_{p_{1}}(i)-\mathcal{O}_{p_{2}}(i)\right)
$$

Note that the bound $H$ is not the same as the one of Proposition 3, because here we iterate over indexes (not over 1s).

\subsection{Sampled Clocks}

The on operator computes the rhythm of a sampled flow. It can express the output clock of the when operator that keeps or suppresses values of a flow of clock $w_{1}$ depending on a condition w2:

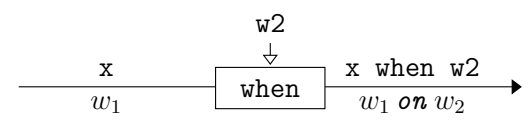

Fig. 4. If $\mathrm{x}$ has clock $w_{1}, \mathrm{x}$ when w2 has clock $w_{1}$ on $w_{2}$. 
Definition 9 (on operator). $0 w_{1}$ on $w_{2} \stackrel{\text { def }}{=} 0\left(w_{1}\right.$ on $\left.w_{2}\right)$

$$
\begin{aligned}
& 1 w_{1} \text { on } 1 w_{2} \stackrel{\text { def }}{=} 1\left(w_{1} \text { on } w_{2}\right) \\
& 1 w_{1} \text { on } 0 w_{2} \stackrel{\text { def }}{=} 0\left(w_{1} \text { on } w_{2}\right)
\end{aligned}
$$

For example, if $w_{1}=11010111 \ldots$ and $w_{2}=101100 \ldots$, then $w_{1}$ on $w_{2}=10010100 \ldots$ Consider the sampling of a flow $\mathrm{x}$ with clock $w_{1}$ by a condition w2:

\begin{tabular}{|c|c|}
\hline \multicolumn{2}{|r|}{$\begin{array}{lllllll}11 & 1 & 0 & 1 & 1 & 1\end{array}$} \\
\hline$w_{2}$ & 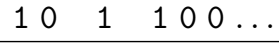 \\
\hline$w_{1}$ on $w_{2}$ & 10010 \\
\hline
\end{tabular}

\begin{tabular}{l|lllllll}
$\mathrm{x}$ & 2 & 5 & 3 & 7 & 9 & 4 & $\ldots$ \\
w2 & 1 & 0 & 1 & 1 & 0 & 0 & $\ldots$ \\
\hline $\mathrm{x}$ when w2 & 2 & 3 & 7 & $\ldots$
\end{tabular}

At each instant, if $\mathbf{x}$ is present, that is, the corresponding element of $w_{1}$ is equal to 1 , the next element of the sampling condition $w_{2}$ is considered. If this element is 1 , then the value of $\mathbf{x}$ is sampled and the flow $\mathrm{x}$ when $\mathrm{w} 2$ is present $\left(w_{1}\right.$ on $w_{2}$ equals 1 ). If the element is 0 , the value of $\mathrm{x}$ is not sampled and the flow $\mathrm{x}$ when w2 is absent $\left(w_{1}\right.$ on $w_{2}$ equals 0$)$. If $\mathbf{x}$ is absent ( $w_{1}$ equals 0$)$, the sampling condition $w_{2}$ is not considered, and the flow $\mathbf{x}$ when w2 will be absent $\left(w_{1}\right.$ on $w_{2}$ equals 0 ).

To compute the on operator on two ultimately periodic binary words $p_{1}$ and $p_{2}$, we first compute the size of the expected result, i.e., $\mid\left(p_{1}\right.$ on $\left.p_{2}\right) . u \mid$, the size of the prefix, and $\mid\left(p_{1}\right.$ on $\left.p_{2}\right) \cdot v \mid$, the size of the periodic part. Then, we compute the value of the elements of the prefix and the periodic part by applying Definition 9.

Proposition 5 (computation of $p_{1}$ on $\left.p_{2}\right)$. Let $p_{1}=u_{1}\left(v_{1}\right)$ and $p_{2}=u_{2}\left(v_{2}\right)$. Then $p_{1}$ on $p_{2}=u_{3}\left(v_{3}\right)$ with: $\left|u_{3}\right|=\max \left(\left|u_{1}\right|, \mathcal{I}_{p_{1}}\left(\left|u_{2}\right|\right)\right)$

$$
\left|v_{3}\right|=\frac{\operatorname{lcm}\left(\left|v_{1}\right|_{1}\left|v_{2}\right|\right)}{\left|v_{1}\right|_{1}} \times\left|v_{1}\right|
$$

and $\forall i, 1 \leq i \leq\left|u_{3}\right|, \quad u_{3}[i]=\left(p_{1}\right.$ on $\left.p_{2}\right)[i]$

$$
\forall i, 1 \leq i \leq\left|v_{3}\right|, \quad v_{3}[i]=\left(p_{1} \text { on } p_{2}\right)\left[\left|u_{3}\right|+i\right]
$$

Intuitively, the prefix of $p_{1}$ on $p_{2}$ is obtained after completely processing the prefixes of $p_{1}$ and $p_{2}$. One element of $p_{1}$ is processed to produce one element of $p_{1}$ on $p_{2}$. Thus, the processing of the elements of $p_{1} . u$ for the computation of the on terminates at the index $\left|p_{1} \cdot u\right|$. An element of $p_{2}$ is processed only when there is a 1 in $p_{1}$. Thus the processing of the elements of $p_{2} . u$ terminates at the index $\mathcal{I}_{p_{1}}\left(\left|p_{2} . u\right|\right)$. Therefore, the size of the prefix of $p_{1}$ on $p_{2}$ is the maximum of $\left|p_{1} . u\right|$ and $\mathcal{I}_{p_{1}}\left(\left|p_{2} . u\right|\right)$. The size of the periodic pattern is obtained by the computation of the common period of $p_{1}$ and $p_{2}$ when $p_{2}$ is processed at the rhythm of the 1s of $p_{1}$.

The result of the on operation can be computed more simply for certain shapes of arguments. The simplest case is the one where the number of $1 \mathrm{~s}$ in the prefix and in the periodic pattern of the first word are, respectively, equal to the size of the prefix and the size of the periodic pattern of the second word as in the following example:

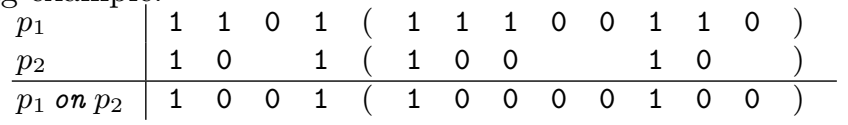


Proposition 6. Consider $p_{1}$ and $p_{2}$ such that $\left|p_{1} \cdot u\right|_{1}=\left|p_{2} \cdot u\right|$ and $\left|p_{1} \cdot v\right|_{1}=\left|p_{2} \cdot v\right|$. Then:

$$
\begin{aligned}
\mid\left(p_{1} \text { on } p_{2}\right) \cdot u \mid & =\left|p_{1} \cdot u\right| & & \mid\left.\left(p_{1} \text { on } p_{2}\right) \cdot u\right|_{1}=\left|p_{2} \cdot u\right|_{1} \\
\mid\left(p_{1} \text { on } p_{2}\right) \cdot v \mid & =\left|p_{1} \cdot v\right| & & \mid\left.\left(p_{1} \text { on } p_{2}\right) \cdot v\right|_{1}=\left|p_{2} \cdot v\right|_{1}
\end{aligned}
$$

As explained in Remark 4, it is possible to increase the size and the number of $1 \mathrm{~s}$ in the prefixes and periodic patterns of words. Therefore, we can always adjust the operands of the on such that they satisfy the assumptions of Proposition 6 . Proposition 6 can be generalized to the case where the number of $1 \mathrm{~s}$ of $p_{1}$ is increased by any multiple of the size of $p_{2} . v$.

Proposition 7. Consider $p_{1}$ and $p_{2}$ such that $\left|p_{1} \cdot u\right|_{1}=\left|p_{2} \cdot u\right|+k \times\left|p_{2} \cdot v\right|$ and $\left|p_{1} . v\right|_{1}=k^{\prime} \times\left|p_{2} . v\right|$ with $k \in \mathbb{N}$ and $k^{\prime} \in \mathbb{N}-\{0\}$. Then:

$$
\begin{aligned}
& \mid\left.\left(p_{1} \text { on } p_{2}\right) \cdot u|=| p_{1} \cdot u|\quad|\left(p_{1} \text { on } p_{2}\right) \cdot u\right|_{1}=\left|p_{2} \cdot u\right|_{1}+k \times\left|p_{2} \cdot v\right|_{1} \\
& \mid\left.\left(p_{1} \text { on } p_{2}\right) \cdot v|=| p_{1} \cdot v|\quad|\left(p_{1} \text { on } p_{2}\right) \cdot v\right|_{1}=k^{\prime} \times\left|p_{2} \cdot v\right|_{1}
\end{aligned}
$$

Finally, to compute the index of the $j$ th 1 of $w_{1}$ on $w_{2}\left(\mathcal{I}_{w_{1} \text { on } w_{2}}(j)\right)$, there is no need to compute the word $w_{1}$ on $w_{2}$ and then to apply the $\mathcal{I}$ function since it can be computed directly from $\mathcal{I}_{w_{1}}$ and $\mathcal{I}_{w_{2}}$.

Proposition 8 (index of the $j$ th 1 of $w_{1}$ on $w_{2}$ ).

$$
\forall j \geq 1, \mathcal{I}_{w_{1} \text { on } w_{2}}(j)=\mathcal{I}_{w_{1}}\left(\mathcal{I}_{w_{2}}(j)\right)
$$

Indeed, in the computation of $w_{1}$ on $w_{2}$, the elements of $w_{2}$ are given when there is a 1 in $w_{1}$. Therefore the index of the $i$ th element of $w_{2}$ is at index $\mathcal{I}_{w_{1}}(i)$. Since the $1 \mathrm{~s}$ of $w_{1}$ on $w_{2}$ are the $1 \mathrm{~s}$ of $w_{2}$, the $j$ th 1 of $w_{1}$ on $w_{2}$ is the $\mathcal{I}_{w_{2}}(j)$ th element of $w_{2}$ and thus at the index $\mathcal{I}_{w_{1}}\left(\mathcal{I}_{w_{2}}(j)\right)$.

We now have all the algebraic tools needed to define an algorithm for the resolution of adaptability constraints on ultimately periodic binary words.

\section{Adaptability Constraints Resolution Algorithm}

We saw in Section 3 that subtyping constraints can be reduced to adaptability constraints where the unknowns are no longer types but rather ultimately periodic binary words. This is the first step of the subtyping constraints resolution algorithm which is summarized in Figure $5 .^{7}$ This section details the remaining steps.

In Section 5.1, we explain how to simplify an adaptability constraint system (S2) to obtain a system (S3) where all the adaptability constraints have the form $c_{x}$ on $p_{x}<: c_{y}$ on $p_{y}$. In Section 5.2, we explain the transformation of adaptability constraints (S3) into a system (S6) of linear inequalities where the unknowns are the size and the indexes of 1s of the sought words. This last system can be solved using standard techniques from Integer Linear Programming, and the resulting solutions can be used to reconstruct the unknown words. In Section 5.3, we discuss the choice of the objective function for the resolution

\footnotetext{
7 A detailed and commented implementation of the algorithm in OCaml is provided at http://www.lri.fr/ mandel/mpc12.
} 


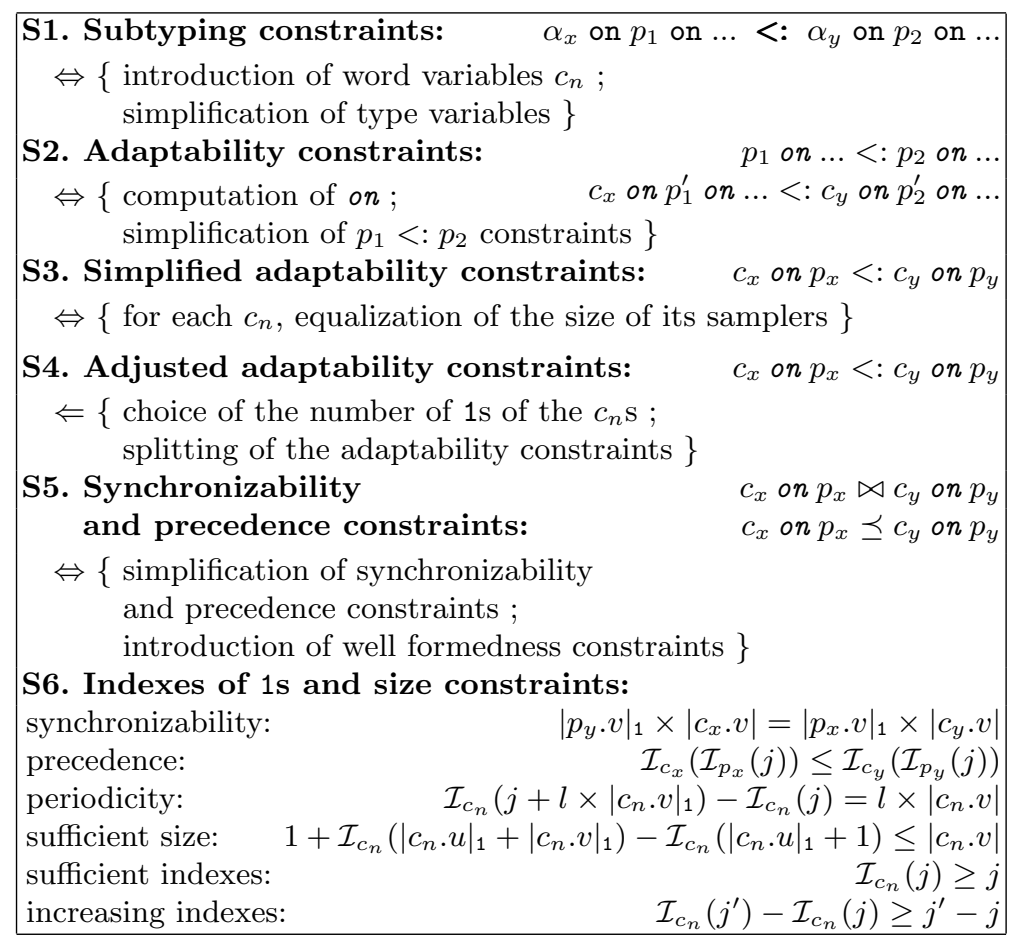

Fig. 5. Summary of the subtyping constraints resolution algorithm. The form of the constraints is given for each system.

of the linear inequalities. Finally, we discuss the correctness, completeness and complexity of the algorithm.

Before the detailed explanation of the algorithm, we note that, as was the case for the equality constraints, there is no greedy algorithm for solving adaptability constraints. Indeed, if the words $\left(c_{1}, c_{2}\right)$ satisfy a constraint $c_{1}$ on $p_{1}<: c_{2}$ on $p_{2}$, then $\left(1^{d} c_{1}, 1^{d} c_{2}\right)$ and $\left(0^{d} c_{1}, 0^{d} c_{2}\right)$ also satisfy it whatever $d$ is. Hence, contrary to a classical subtyping system, we cannot simply take the greatest word for a variable on the left of an adaptability constraint, and the smallest one for a variable on the right. In our case, the inference of values satisfying constraints must necessarily be performed globally to choose words big enough, and/or small enough to satisfy all constraints.

\subsection{Constraint System Simplification}

We begin by considering adaptability constraint systems. After the computation of on operators (Proposition 5), these systems comprise constraints of the form $p_{x}<: p_{y}$ and $c_{x}$ on $p_{x}<: c_{y}$ on $p_{y}$, where $p_{x}$ and $p_{y}$ are known words of non-null rate and $c_{x}$ and $c_{y}$ are unknown words. 
Since a constraint of the form $p_{x}<: p_{y}$ contains no variables, its truth value cannot be altered. We need only to check that each such constraint is satisfied, which is done by applying Definition 7 . If any are false, then the whole system is unsatisfiable. The true constraints can be removed from the system.

Returning to the cyclic_encoding example, the adaptability constraint system was:

$$
\left\{\begin{array}{l}
\left(0^{50} 100\right)<:\left(0^{50} 100\right) \\
\left(0^{50} 100\right)<:\left(0^{50} 010\right) \\
\left(0^{50} 100\right)<:\left(0^{50} 001\right)
\end{array}\right\}
$$

Through the application of the adaptability test, we can check that each constraint is always satisfied. Here, after simplification, the system is empty and thus the node cyclic_encoding is well typed.

In the general case, after simplification all the remaining constraints contain variables. For example, the subtyping constraint system $C^{\prime}$ of Section 3 can be rewritten to the adaptability constraint system $A^{\prime}$ :

$$
\theta\left(C^{\prime}\right) \Leftrightarrow\left\{\begin{aligned}
& c_{1} \text { on } 10(1)<: c_{2} \text { on }(01) \\
&(1100)<:(01) \\
& c_{2} \text { on }(01) \text { on }(10)<: c_{3} \text { on }(1) \\
& c_{2} \text { on }(01) \text { on }(01)<: c_{3} \text { on }(1)
\end{aligned}\right\} \Leftrightarrow\left\{\begin{array}{l}
c_{1} \text { on } 10(1)<: c_{2} \text { on }(01) \\
c_{2} \text { on }(0100)<: c_{3} \text { on }(1) \\
c_{2} \text { on }(0001)<: c_{3} \text { on }(1)
\end{array}\right\}=A^{\prime}
$$

All the remaining constraints are of the form $c_{x}$ on $p_{x}<: c_{y}$ on $p_{y}$.

\subsection{Constraint System Solving}

The goal now is to solve adaptability constraint systems of the form:

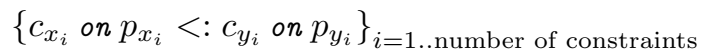

The values $p_{x_{i}}, p_{y_{i}}$ are some known ultimately periodic binary words of nonnull rate. The variables $c_{x_{i}}, c_{y_{i}}$ are the unknowns of the system. Note that some unknown variables can appear several times in a system like in $A^{\prime}$ (we only know that $c_{x_{i}} \neq c_{y_{i}}$ since simplification has been performed): ${ }^{8}$

$$
\left\{\begin{array}{l}
c_{1} \text { on } p_{1}<: c_{2} \text { on } p_{2} \\
c_{2} \text { on } p_{2}^{\prime}<: c_{3} \text { on } p_{3} \\
c_{2} \text { on } p_{2}^{\prime \prime}<: c_{3} \text { on } p_{3}^{\prime}
\end{array}\right\}
$$

Solving the system means associating ultimately periodic words of non-null rate to the unknowns $\left(c_{1}, c_{2}, c_{3}\right)$, such that the constraints are satisfied.

Remark \%. If solutions containing null rates are allowed, all systems have a solution. For example, the instantiation $\forall n . c_{n}=(0)$ is a trivial solution of all systems. A solution containing a null rate gives a system that will be executed at only a finite number of instants. We are not interested in such solutions.

An adaptability constraint $c_{x}$ on $p_{x}<: c_{y}$ on $p_{y}$ can be decomposed into a synchronizability constraint and a precedence constraint:

$$
\begin{aligned}
c_{x} \text { on } p_{x}<: c_{y} \text { on } p_{y} \Leftrightarrow & \{\text { by Definition } 7\} \\
& \left(c_{x} \text { on } p_{x} \bowtie c_{y} \text { on } p_{y}\right) \wedge\left(c_{x} \text { on } p_{x} \preceq c_{y} \text { on } p_{y}\right)
\end{aligned}
$$

\footnotetext{
${ }^{8}$ We choose the same index for a variable $c_{n}$ and the words $p_{n}, p_{n}^{\prime}, \ldots$ that sample $c_{n}$.
} 
The synchronizability constraint can itself be rewritten:

$$
\begin{aligned}
c_{x} \text { on } p_{x} \bowtie c_{y} \text { on } p_{y} \Leftrightarrow & \{\text { by Proposition } 2 \text { and Proposition } 1\} \\
& \frac{\mid\left.\left(c_{x} \text { on } p_{x}\right) \cdot v\right|_{1}}{\mid\left(c_{x} \text { on } p_{x}\right) \cdot v \mid}=\frac{\mid\left.\left(c_{y} \text { on } p_{y}\right) \cdot v\right|_{1}}{\mid\left(c_{y} \text { on } p_{y}\right) \cdot v \mid}
\end{aligned}
$$

As can the precedence constraint:

$$
\begin{gathered}
c_{x} \text { on } p_{x} \preceq c_{y} \text { on } p_{y} \Leftrightarrow\{\text { by Proposition } 3 \text { and Proposition } 8\} \\
\qquad j, 1 \leq j \leq h, \mathcal{I}_{c_{x}}\left(\mathcal{I}_{p_{x}}(j)\right) \leq \mathcal{I}_{c_{y}}\left(\mathcal{I}_{p_{y}}(j)\right) \\
\text { with } h=\max \left(\mid\left.\left(c_{x} \text { on } p_{x}\right) \cdot u\right|_{1}, \mid\left.\left(c_{y} \text { on } p_{y}\right) \cdot u\right|_{1}\right)+\operatorname{lcm}\left(\mid\left.\left(c_{x} \text { on } p_{x}\right) \cdot v\right|_{1}, \mid\left.\left(c_{y} \text { on } p_{y}\right) \cdot v\right|_{1}\right)
\end{gathered}
$$

We are thus interested in the size of, and the number of $1 \mathrm{~s}$ in, the prefixes and periodic patterns of $c_{x}$ on $p_{x}$ and of $c_{y}$ on $p_{y}$ forms.

We have seen in Section 4 (Proposition 7) that the size and number of $1 \mathrm{~s}$ in the prefix and in the periodic pattern of $c_{n}$ on $p_{n}$ can easily be expressed as a function of the size and number of $1 \mathrm{~s}$ in the prefixes and periodic patterns of $c_{n}$ and $p_{n}$ in the following case: $\left|c_{n} . u\right|_{1}=\left|p_{n} \cdot u\right|+k \times\left|p_{n} \cdot v\right|$ and $\left|c_{n} \cdot v\right|_{1}=k^{\prime} \times\left|p_{n} . v\right|$.

To put the system into this "simple" form, we adjust the known words of the system to satisfy the property: for any unknown $c_{n}$, all its samplers ${ }^{9} p_{n}$, $p_{n}^{\prime}, \ldots$ have the same prefix size $\left(\left|p_{n} . u\right|=\left|p_{n}^{\prime} . u\right|=\ldots\right)$ and the same periodic pattern size $\left(\left|p_{n} \cdot v\right|=\left|p_{n}^{\prime} \cdot v\right|=\ldots\right)$. This operation is always possible (thanks to Remark 4) and does not change the semantics of the system.

We can then choose the number of $1 \mathrm{~s}$ in the unknown $c_{n}$.

Choice 1 (number of $1 \mathrm{~s}$ in the $c_{n}$ ) Let $k \in \mathbb{N}$ and $k^{\prime} \in \mathbb{N}-\{0\}$.

$$
\begin{array}{ll}
\left|c_{n} \cdot u\right|_{1}=\left|p_{n} \cdot u\right|+k \times\left|p_{n} \cdot v\right| & \left(=\left|p_{n}^{\prime} \cdot u\right|+k \times\left|p_{n}^{\prime} \cdot v\right|=\ldots\right) \\
\left|c_{n} \cdot v\right|_{1}=k^{\prime} \times\left|p_{n} \cdot v\right| & \left(=k^{\prime} \times\left|p_{n}^{\prime} \cdot v\right|\right.
\end{array}
$$

where $p_{n}, p_{n}^{\prime}, \ldots$ are the samplers of $c_{n}$.

Remark 8. The algorithm is parameterized by constants $k$ and $k^{\prime}$, which restrict the number of $1 \mathrm{~s}$ in any solution and may thus lead to failures in the resolution of constraints. We will discuss this choice in Section 5.4.

This choice allows us to express the size and the number of $1 \mathrm{~s}$ in the prefixes and periodic patterns of the $c_{n}$ on $p_{n}$ in terms of those of $c_{n}$ and $p_{n}$. Hence, a synchronizability constraint becomes:

$$
\begin{aligned}
& c_{x} \text { on } p_{x} \bowtie c_{y} \text { on } p_{y} \Leftrightarrow\{\text { by Proposition } 7\} \\
& \frac{k^{\prime} \times\left|p_{x} \cdot v\right|_{1}}{\left|c_{x} \cdot v\right|}=\frac{k^{\prime} \times\left|p_{y} \cdot v\right|_{1}}{\left|c_{y} \cdot v\right|} \\
& \Leftrightarrow\left|p_{y} \cdot v\right|_{1} \times\left|c_{x} \cdot v\right|=\left|p_{x} \cdot v\right|_{1} \times\left|c_{y} \cdot v\right|
\end{aligned}
$$

And a precedence constraint becomes:

$$
\begin{gathered}
c_{x} \text { on } p_{x} \preceq c_{y} \text { on } p_{y} \Leftrightarrow\{\text { by Proposition } 7\} \\
\forall j, 1 \leq j \leq h, \mathcal{I}_{c_{x}}\left(\mathcal{I}_{p_{x}}(j)\right) \leq \mathcal{I}_{c_{y}}\left(\mathcal{I}_{p_{y}}(j)\right) \\
\text { with } h=\max \left(\left|p_{x} \cdot u\right|_{1}+k \times\left|p_{x} . v\right|_{1},\left|p_{y} \cdot u\right|_{1}+k \times\left|p_{y} \cdot v\right|_{1}\right)+ \\
\operatorname{lcm}\left(k^{\prime} \times\left|p_{x} \cdot v\right|_{1}, k^{\prime} \times\left|p_{y} \cdot v\right|_{1}\right)
\end{gathered}
$$

\footnotetext{
${ }^{9} \mathrm{~A}$ word $p_{n}$ is a sampler of $c_{n}$ if $c_{n}$ on $p_{n}$ is in the constraint system.
} 
For example, we can adjust the system $A^{\prime}$ such that all the samplers of a particular variable have the same size:

$$
A^{\prime}=\left\{\begin{array}{l}
c_{1} \text { on } 10(1)<: c_{2} \text { on }(01) \\
c_{2} \text { on }(0100)<: c_{3} \text { on }(1) \\
c_{2} \text { on }(0001)<: c_{3} \text { on }(1)
\end{array}\right\} \Leftrightarrow\left\{\begin{array}{l}
c_{1} \text { on } 10(1)<: c_{2} \text { on }(0101) \\
c_{2} \text { on }(0100)<: c_{3} \text { on }(1) \\
c_{2} \text { on }(0001)<: c_{3} \text { on }(1)
\end{array}\right\}
$$

Then, we choose the number of $1 \mathrm{~s}$ for the $c_{n} \mathrm{~s}$ to be equal to the size of the respective samplers:

$$
\begin{array}{lll}
\left|c_{1} \cdot u\right|_{1}=2+k \times 1 & \left|c_{2} \cdot u\right|_{1}=0+k \times 4 & \left|c_{3} \cdot u\right|_{1}=0+k \times 1 \\
\left|c_{1} \cdot v\right|_{1}=k^{\prime} \times 1 & \left|c_{2} \cdot v\right|_{1}=k^{\prime} \times 4 & \left|c_{3} \cdot v\right|_{1}=k^{\prime} \times 1
\end{array}
$$

By Formula (1), the synchronizability constraints become a system of linear equations on the size of the periodic patterns of the $c_{n} \mathrm{~s}$ :

$$
\left\{\begin{aligned}
|(0101) \cdot v|_{1} \times\left|c_{1} \cdot v\right| & =|(10(1)) \cdot v|_{1} \times\left|c_{2} . v\right| \\
|(1) . v|_{1} \times\left|c_{2} . v\right| & =|(0100) \cdot v|_{1} \times\left|c_{3} . v\right| \\
|(1) . v|_{1} \times\left|c_{2} . v\right| & =|(0001) \cdot v|_{1} \times\left|c_{3} . v\right|
\end{aligned}\right\} \Leftrightarrow\left\{\begin{aligned}
2 \times\left|c_{1} \cdot v\right| & =\left|c_{2} . v\right| \\
\left|c_{2} \cdot v\right| & =\left|c_{3} . v\right| \\
\left|c_{2} . v\right| & =\left|c_{3} . v\right|
\end{aligned}\right\}
$$

By Formula (2), if we choose the constants $k$ and $k^{\prime}$ to be equal to 0 and 1 , the precedence constraints become a system of linear inequalities on the indexes of $1 \mathrm{~s}$ in the $c_{n} \mathrm{~s}$ :

$$
\left\{\begin{array}{l}
\forall j, 1 \leq j \leq 3, \quad \mathcal{I}_{c_{1}}\left(\mathcal{I}_{10(1)}(j)\right) \leq \mathcal{I}_{c_{2}}\left(\mathcal{I}_{(0101)}(j)\right) \\
\forall j, 1 \leq j \leq 1, \quad \mathcal{I}_{c_{2}}\left(\mathcal{I}_{(0100)}(j)\right) \leq \mathcal{I}_{c_{3}}\left(\mathcal{I}_{(1)}(j)\right) \\
\forall j, 1 \leq j \leq 1, \quad \mathcal{I}_{c_{2}}\left(\mathcal{I}_{(0001)}(j)\right) \leq \mathcal{I}_{c_{3}}\left(\mathcal{I}_{(1)}(j)\right)
\end{array}\right\}
$$

which is equivalent to the following system after the computation of the $\mathcal{I}_{p_{n}}(j)$ :

$$
\left\{\begin{array}{l}
\mathcal{I}_{c_{1}}(1) \leq \mathcal{I}_{c_{2}}(2) \\
\mathcal{I}_{c_{1}}(3) \leq \mathcal{I}_{c_{2}}(4) \\
\mathcal{I}_{c_{1}}(4) \leq \mathcal{I}_{c_{2}}(6) \\
\mathcal{I}_{c_{2}}(2) \leq \mathcal{I}_{c_{3}}(1) \\
\mathcal{I}_{c_{2}}(4) \leq \mathcal{I}_{c_{3}}(1)
\end{array}\right\}
$$

Now, to give a value to each unknown $c_{n}$, we must find its size (satisfying Sync) and the positions of its 1s (satisfying Prec). Hence, the sizes $\left|c_{n} . v\right|$ and the indexes $\mathcal{I}_{c_{n}}(j)$ will no longer be considered as function applications, but rather as the new unknowns of the problem. These new unknowns must also satisfy the constraints of Remarks 2, 3, 5 and 6 which ensure that the solution will be a well formed ultimately periodic binary word. So, we have to augment Sync and Prec with the following four sets of constraints: ${ }^{10}$

Periodicity: $\operatorname{Per}=\left\{\mathcal{I}_{c_{n}}\left(j+l \times\left|c_{n} . v\right|_{1}\right)-\mathcal{I}_{c_{n}}(j)=l \times\left|c_{n} . v\right|\right\}_{\mathcal{I}_{c_{n}}\left(j+l \times\left|c_{n} . v\right|_{1}\right) \in \text { Prec }}$

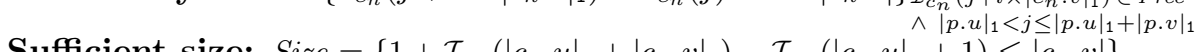

Sufficient size: Size $=\left\{1+\mathcal{I}_{c_{n}}\left(\left|c_{n} . u\right|_{1}+\left|c_{n} . v\right|_{1}\right)-\mathcal{I}_{c_{n}}\left(\left|c_{n} . u\right|_{1}+1\right) \leq\left|c_{n} . v\right|\right\}$

Sufficient indexes: Init $=\left\{\mathcal{I}_{c_{n}}(j) \geq j\right\}_{\mathcal{I}_{c_{n}}(j) \in \text { Prec } \cup \text { Per } \cup \text { Size }}$

Increasing indexes:Incr $=\left\{\mathcal{I}_{c_{n}}\left(j^{\prime}\right)-\mathcal{I}_{c_{n}}(j) \geq j^{\prime}-j\right\}_{\left(\mathcal{I}_{c_{n}}(j), \mathcal{I}_{c_{n}}\left(j^{\prime}\right)\right) \in \text { Prec } \cup \text { Per } \cup \text { Size }}$

Finally, we can use a generic solver for Integer Linear Programming (ILP) problems to solve the system: $S=$ Sync $\cup$ Prec $\cup$ Per $\cup$ Size $\cup$ Init $\cup$ Incr.

$\overline{10}$ The notation $\mathcal{I}_{c_{n}}(j) \in S$ designates the presence of the unknown $\mathcal{I}_{c_{n}}(j)$ in $S$. 
Applying a solver to the system associated with $A^{\prime}$ produces the results:

$$
\begin{array}{lllll}
\left|c_{1} \cdot v\right|=2 & \mathcal{I}_{c_{1}}(1)=1 & \mathcal{I}_{c_{1}}(3)=3 & \mathcal{I}_{c_{1}}(4)=5 & \\
\left|c_{2} \cdot v\right|=4 & \mathcal{I}_{c_{2}}(1)=1 & \mathcal{I}_{c_{2}}(2)=2 & \mathcal{I}_{c_{2}}(4)=4 & \mathcal{I}_{c_{2}}(6)=6 \\
\left|c_{3} \cdot v\right|=4 & \mathcal{I}_{c_{3}}(1)=4 & & &
\end{array}
$$

Thanks to this information and the number of $1 \mathrm{~s}$ in the prefixes and periodic patterns of the $c_{n}$ s chosen previously, we can build the following solution to the $A^{\prime}$ system: $c_{1}=11(10) \quad c_{2}=(1111)=(1) \quad c_{3}=000(1000)=\left(0^{3} 1\right)$.

We now know all the clock types of the system of Figure 3 . The result gives us the clock type of the node $f$ which is $\forall \alpha, \alpha$ on $c_{1} \times \alpha$ on $c_{2} \rightarrow \alpha$ on $c_{3}$, that is: $f:: \forall \alpha, \alpha$ on $11(10) \times \alpha$ on (1) $\rightarrow \alpha$ on $\left(0^{3} 1\right)$.

And since we have the types of the buffers, we can compute their sizes. For example, we know that the writing clock of the first buffer is of type $\alpha$ on $c_{1}$ on 10(1) $=\alpha$ on 11(10) on 10(1) and that the reading clock is of type $\alpha$ on $c_{2}$ on (01) $=\alpha$ on (1) on (01). By Proposition 4, the size of this buffer is: size $(11(10)$ on $10(1),(1)$ on $(01))=1$.

\subsection{Guiding the Resolution Algorithm}

The resolution algorithm requires the solution of linear inequalities on the indexes of $1 \mathrm{~s}$ and on the size of the unknown words. Tools for solving such inequalities are parameterized by an objective function determining the criterion to optimize. In the previous example, we choose to optimize the sum of the indexes of 1 s to produce a kind of As-Soon-As-Possible schedule. But we can also use the objective function to favor either system throughput or buffer sizes minimization. We illustrate this trade-off on an example:
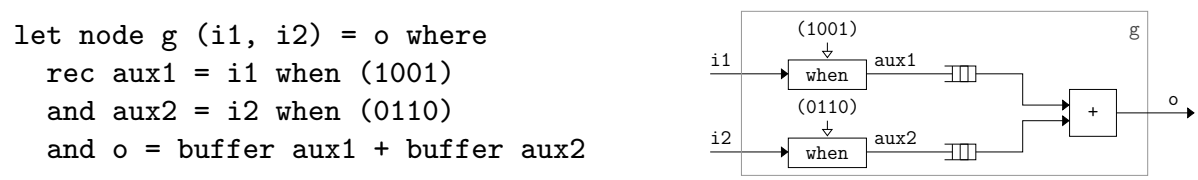

If we assign the type $\forall \alpha .(\alpha \times \alpha) \rightarrow \alpha$ on (01) to the node $\mathrm{g}$, the buffers will be of size 1 . But, this node can be executed without buffers if we give it the type: $g:: \forall \alpha .(\alpha$ on $(011110) \times \alpha$ on (110011) $) \rightarrow \alpha$ on (010010) .

The first solution can be obtained by an objective function that minimizes the size of the solution. Indeed, since the number of $1 \mathrm{~s}$ in the solution is fixed, minimizing the size increases the rate.

The second solution is obtained by an objective function that, for all precedence constraints $\mathcal{I}_{c_{x}}\left(j_{1}\right) \leq \mathcal{I}_{c_{y}}\left(j_{2}\right)$, minimizes the value $\mathcal{I}_{c_{y}}\left(j_{2}\right)-\mathcal{I}_{c_{x}}\left(j_{1}\right)$. It means that we minimize the number of instants between the writing and the reading of a value in a buffer which has the consequence of reducing the buffer sizes.

\subsection{Correctness, Completeness and Complexity}

The resolution algorithm shown in Figure 5 relies on the step-by-step transformation of the adaptability constraint system (S2) into linear inequalities (S6), for which there exist algorithms that find a solution if it exists [20]. Each step, 
except the one between S4 and S5, is a rewriting of a constraint system into an equivalent one (thanks to the equivalence properties stated in Section 4). The step from S4 to S5 is the choice of the number of $1 \mathrm{~s}$ in the $c_{n} \mathrm{~s}$. It is correct to seek a solution in a subset of all possible words. Nevertheless, it may lead to incompleteness, since it is possible that a system has no solution in the subset of words considered.

We have parameterized our resolution algorithm by two constants $k$ and $k^{\prime}$ which modify the number of $1 \mathrm{~s}$ in the sought solution. A semi-decidable algorithm to solve adaptability constraints iterates the previous algorithm with $k=0,1,2, \ldots$ and $k^{\prime}=k+1$ until it finds a solution. We can prove that this algorithm is complete because if a system of adaptability constraints has a solution $S$, then there exists a solution $S^{\prime}$ such that $\forall c_{n}^{\prime} \in S^{\prime}$,

$$
\begin{aligned}
& \left|c_{n}^{\prime} \cdot u\right|_{1}=\left|p_{n} \cdot u\right|+k \times\left|p_{n} \cdot v\right| \quad\left(=\left|p_{n}^{\prime} \cdot u\right|+k \times\left|p_{n}^{\prime} \cdot v\right|=\ldots\right) \\
& \left|c_{n}^{\prime} \cdot v\right|_{1}=(k+1) \times\left|p_{n} \cdot v\right| \quad\left(=(k+1) \times\left|p_{n}^{\prime} \cdot v\right|=\ldots\right)
\end{aligned}
$$

where $k$ can be computed from the original solution $S$. The idea of the proof is to use Remark 4 to rewrite $S$ into $S^{\prime}$ (the detailed proof is in the extended version of the paper).

Moreover, note that the step that equalizes of the size of the samplers (from S3 to S4, Figure 5) can be adapted such that the choice $k=0$ and $k=1$ always leads to a solution, if it exists, for (1) systems that do not have prefixes, (2) systems where the prefixes of the samplers of a variable are made of os and have the same size, and (3) systems with only one constraint. The algorithm is given in the extended version of the paper.

Remark that we can sometimes find solutions that allow faster execution of a system if we choose a number of 1s different than the one proposed by $k=0$ and $k^{\prime}=1$. For example, consider the following adaptability constraints:

$$
\left\{c_{1} \text { on (1) }<: c_{2} \text { on }(110)\right\}
$$

If we are seeking a solution with one 1 for $c_{1}$, we compute the solution $\left\{c_{1}=(10)\right.$; $\left.c_{2}=(1011)\right\}$ where rate $\left(c_{1}\right)=\frac{1}{2}$ and $\operatorname{rate}\left(c_{2}\right)=\frac{3}{4}$. Whereas, if we are seeking a solution with two $1 \mathrm{~s}$ for $c_{1}$, we compute the solution $\left\{c_{1}=(110) ; c_{2}=\left(1^{6}\right)\right\}$ where rate $\left(c_{1}\right)=\frac{2}{3}$ and $\operatorname{rate}\left(c_{2}\right)=1$. The guarantee provided by the resolution algorithm is that for a given number of $1 \mathrm{~s}$, the result is optimal with respect to the objective function given to the ILP solver. It follows the fact that each transformation of the adaptability constraint system, except Choice 1, maintains equivalence. Therefore, there is no loss of information.

The complexity of the resolution algorithm is dominated by the resolution of the constraint system on the indexes of 1s and the sizes. This is an ILP problem which is known to be NP-complete [20]. Even if there is only one adaptability constraint per buffer, the size of the complete ILP problem can be big (e.g., millions of variables): it depends on the size of the samplers in the adaptability constraint system. 


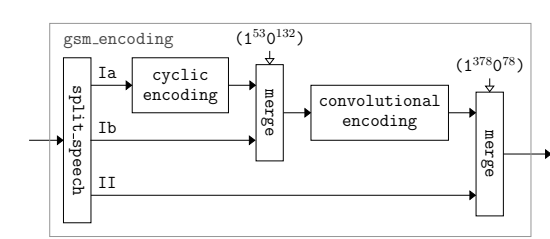

(a) GSM speech encoder.

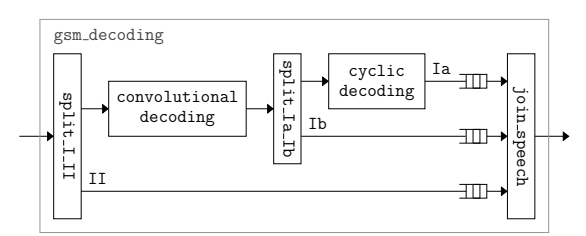

(b) GSM speech decoder.

In the GSM protocol [13], speech is divided into samples of $20 \mathrm{~ms}$, each of which is encoded as a frame of 260 bits. These frames are then encoded, such that it is possible to detect and sometimes to correct errors introduced by radio transmission.

The 260 bits are divided into three classes: Ia, Ib, II. Class Ia contains the first 50 and most important bits, class Ib contains the following 132 moderately important bits and class II contains the last and least important 78 bits. The 50 bits of class Ia go through the cyclic encoder that is presented in Section 2. Then the 132 bits of class Ib are appended to the 50 bits of class Ia followed by the 3 cyclic redundancy bits. The 185 bits thus obtained are encoded by a convolution encoder which builds packets of 378 bits, to which the 78 bits of class II are appended.

The decoder has the reverse behavior. It starts by separating the bits of class I from the bits of class II that have not been encoded. The bits of class I are then processed by a convolution decoder that detects and corrects some errors. Finally, a cyclic decoder is applied to the bits of class Ia to check that there are no errors (if errors remain, the bits of the preceding frame are transmitted).

Fig. 6. Excerpt of the GSM speech encoder/decoder.

\section{Comparison with Previous Resolution Algorithms}

Three algorithms for the resolution of adaptability constraints have been proposed. The first one [8] is based on the successive application of local simplification rules. This algorithm does not always succeed because some systems can only be simplified globally, that is, by resolving all of their constraints simultaneously (one such example is given in the long version of this article).

A second algorithm [17], the abstract resolution algorithm, is based on the abstraction of clocks by sets of clocks defined by an asymptotic rate and two offsets bounding the potential delay with respect to this rate [9]. Thanks to this abstraction, the adaptability relation can be tested by some simple operations on rational numbers.

Section 5 of this article presents the third resolution algorithm, the concrete resolution algorithm. Technically, the first steps of the algorithm (from S1 to S3, Figure 5) are similar to the ones of the abstract resolution algorithm. The subsequent steps that solve the adaptability constraints (from S3 to S6) differ. In the rest of the section, we will focus on the comparison of the concrete resolution algorithm and the abstract resolution algorithm via some specific examples.

The concrete resolution algorithm allows us to type an excerpt of a GSM speech encoder/decoder. The principle of the encoder/decoder is described in Figure 6 and the source code is available at http://www.lri.fr/ mandel/ mpc12. This example illustrates the advantages of concrete resolution.

The encoder is depicted in Figure 6(a). The different nodes contain buffers, but they are connected without buffers. The global unification mentioned in 
Remark 1 is essential to type this node. It can find a rhythm for consuming the input flow such that all the constraints imposed by the processing of the three branches are satisfied.

The abstract resolution algorithm cannot type this node because it cannot treat a unification constraint as a pair of inverse subtyping constraints as proposed in Remark 1. Indeed, when clocks are abstracted, we do not have sufficiently precise information about them to guarantee equality. So, to type the gsm_encoding node with the abstract resolution algorithm, we would have to add buffers to communicate the values of the flows Ia, Ib and II, which would transform the unification constraints into subtyping constraints which could be solved. With this new version of the gsm_encoding node, the buffer sizes estimated by abstract resolution are 50, 132 and 78, whereas the concrete resolution showed that, for the same throughput, such buffers are not necessary.

The GSM encoder example shows that the concrete resolution algorithm can handle programs that need subtle node scheduling. This advantage is also evident in programs that contain cycles with few initialization values such as the following one:

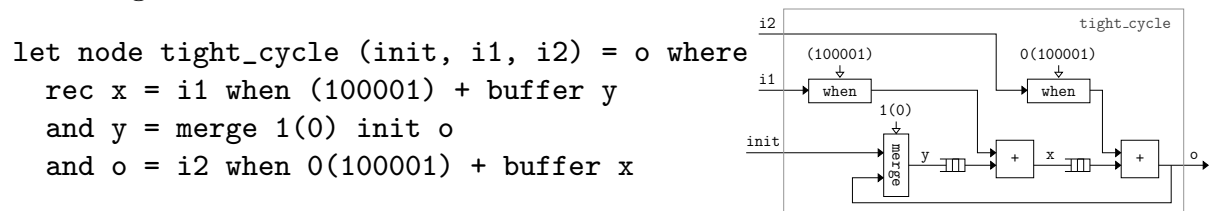

Here, since there is only one initial value in the cycle, the activations of the two + operators are tightly coupled: they must alternate. The abstract resolution algorithm cannot find such a schedule because, in this case, due to the lost information, it cannot guarantee safe communication through the buffers. The concrete resolution algorithm, on the other hand, finds a correct schedule.

Let us now consider the GSM speech decoder depicted in Figure 6(b). Notice that the flows Ia, Ib are II are buffered. The concrete resolution algorithm infers buffer sizes of, respectively, 1, 132 and 156, while the abstract resolution algorithm gives 51,264 and 234. The buffer sizes estimated by the abstract resolution algorithm are almost twice as large as those found by the concrete algorithm.

This example shows that when buffers are necessary, the concrete resolution algorithm can give better estimates of buffer sizes.

Notice, however, that the abstract resolution algorithm is still interesting for cases like the video application Picture in Picture [17]. Running the concrete resolution algorithm on this example takes several days of computer time! Indeed, because the size of the clock words involved in the system are on the order of two million bits, our algorithm generates a system of linear inequalities containing numbers of variables and constraints of the same order of magnitude. Constraint systems of this size cannot be handled efficiently with tools like GLPK [12] that solve systems of linear inequalities. Finally, the algorithm with abstraction can handle systems where some words are not exactly periodic [9], that is, those with some jitters. 
For a given program, one algorithm may be more appropriate than the other. When the periodic words are well balanced, i.e., when the 1s are regularly spread (as is the case for the nodes of the Picture in Picture application), the algorithm with abstraction gives good results quickly. However, it fails when there are some constraints that are difficult to satisfy: e.g. those requiring global unification or those containing cycles. When words are not well balanced, i.e., when the 1s come in bursts (as in the GSM example) and they are not too long (only hundreds of elements), then the concrete algorithm is better: there is less risk of rejecting a system that has a solution, and the buffer size estimates are better. Finally, unlike the abstract algorithm, the concrete algorithm is not limited to optimizing system throughput. For example, it can find a schedule for Picture in Picture that reduces throughput in order to avoid buffering.

\section{Conclusion}

In this article, we have presented an algorithm that computes schedules and buffer sizes for networks of ultimately periodic processes described as Lucy-n programs.

Scheduling and finding buffer sizes for networks of processes is an old problem. Our particularity is to work in the context of a programming language. In that respect, the most related approaches are those of Ptolemy [11] and StreamIt [22] which are implementations of the Synchronous Data-Flow model [14]. In Ptolemy, the computation nodes are programmed in a host language and the production and consumption rates of nodes are declared by the user. If the values declared by the user are not correct, a program will fail at run-time. The approach of Lucy-n is different: the whole program is written in a single language and the production and consumption rates are inferred automatically from the source code. StreamIt follows the same approach as Lucy-n, but provides only a small number of combinators which restricts the set of networks that can be described.

The main contribution of this paper is to define a resolution algorithm of subtyping constraints that uses all the information contained in the types. Therefore, it can accept more programs than previous algorithms and it does not overestimate buffer sizes.

Even if the algorithm presented in this paper is computationally more complex than the abstract algorithm presented in [17], our new algorithm can type some programs that would be impossible to type with the other one. In particular, the concrete resolution algorithm has been used [18] to type programs that model latency insensitive design [3]. The types that are obtained for the different nodes of such programs define static schedules for the modeled circuit $[7,2,4]$. Because of their shape, all these programs make the abstract algorithm fail.

Finally, a great advantage of the concrete resolution algorithm presented in this article is that it does not restrict the trade-off between buffering and throughput. A direction for future work is to provide new language constructs to declare resource constraints and to use them to guide the resolution algorithm. 
Acknowledgments. First we would like to thank Timothy Bourke for his numerous and useful comments on the article. It has been a great pleasure to interact with him. We are grateful to Gwenaël Delaval for providing the GSM example which is ideal for motivating and illustrating our approach. Marc Pouzet has been very supportive and always has good advice. Last but not least, we would like to thank the reviewers and the MPC program committee for the quality and the benevolence of their remarks.

\section{References}

1. G. Bilsen, M. Engels, R. Lauwereins, and J. Peperstraete. Cyclo-static dataflow. IEEE Trans. on Signal Processing, 44(2):397-408, 1996.

2. J. Boucaron, R. de Simone, and J.-V. Millo. Formal methods for scheduling of latency-insensitive designs. EURASIP Journal on Embedded Systems, Issue 1, January 2007.

3. L. Carloni, K. McMillan, and A. Sangiovanni-Vincentelli. Theory of latencyinsensitive design. IEEE Trans. on CAD of Integrated Circuits ans Systems, 20(9):1059-1076, September 2001.

4. J. Carmona, J. Júlvez, J. Cortadella, and M. Kishinevsky. Scheduling synchronous elastic designs. In Application of Concurrency to System Design, 2009.

5. P. Caspi, D. Pilaud, N. Halbwachs, and J. A. Plaice. Lustre: a declarative language for real-time programming. In Principles of Programming Languages, 1987.

6. P. Caspi and M. Pouzet. Synchronous Kahn networks. In International Conference on Functional Programming, May 1996.

7. M. Casu and L. Macchiarulo. A new approach to latency insensitive design. In Design Automation Conference, 2004.

8. A. Cohen, M. Duranton, C. Eisenbeis, C. Pagetti, F. Plateau, and M. Pouzet. Nsynchronous Kahn networks: a relaxed model of synchrony for real-time systems. In Principles of Programming Languages, 2006.

9. A. Cohen, L. Mandel, F. Plateau, and M. Pouzet. Abstraction of clocks in synchronous data-flow systems. In ASIAN Symposium on Programming Languages and Systems, 2008.

10. J.-L. Colaço and M. Pouzet. Clocks as first class abstract types. In International Conference on Embedded Software, 2003.

11. J. Eker, J.W. Janneck, E.A. Lee, Jie Liu, Xiaojun Liu, J. Ludvig, S. Neuendorffer, S. Sachs, and Yuhong Xiong. Taming heterogeneity - the Ptolemy approach. Proceedings of the IEEE, 91(1):127 - 144, jan 2003.

12. GLPK. GNU linear programming kit. http://www.gnu.org/software/glpk/.

13. X. Lagrange, P. Godlewski, and S. Tabbane. Réseaux GSM : des principes à la norme. Hermès Science, Paris, 2000.

14. Ed. Lee and D. Messerschmitt. Synchronous data flow. IEEE Transactions on Computers, 75(9), September 1987.

15. L. Mandel and F. Plateau. Typage des horloges périodiques en Lucy-n. In Journées Francophones des Langages Applicatifs, La Bresse, France, January 2011.

16. L. Mandel and F. Plateau. Scheduling and buffer sizing of n-synchronous systems - extended version. Available at http://www.lri.fr/ mandel/mpc12, 2012.

17. L. Mandel, F. Plateau, and M. Pouzet. Lucy-n: a n-synchronous extension of Lustre. In Mathematics of Program Construction, 2010. 
18. L. Mandel, F. Plateau, and M. Pouzet. Static scheduling of latency insensitive designs with Lucy-n. In Formal Methods in Computer Aided Design, 2011.

19. W. Wesley Peterson. Error-Correcting Codes. The M.I.T. Press, 1961.

20. A. Schrijver. Theory of linear and integer programming. John Wiley \& Sons, 1986.

21. T. Schrijvers, S. Peyton Jones, M. Sulzmann, and D. Vytiniotis. Complete and decidable type inference for GADTs. In International Conference on Functional Programming, pages 341-352, 2009.

22. W. Thies, M. Karczmarek, and S. Amarasinghe. StreamIt: A language for streaming applications. In International Conference on Compiler Construction, 2002.

\section{A Clock constraints collection}

Clock types are separated into three categories: type schemes $(\sigma)$ which represent the types of nodes, types of expressions $(t)$ and types of streams $(c t)$.

$$
\begin{aligned}
& \sigma::=\forall \beta_{1}, \ldots, \beta_{m} . \forall \alpha_{1}, \ldots, \alpha_{n} . c t \rightarrow c t \\
& t::=\beta|t \times t| c t \\
& c t::=\alpha \mid c t \text { on } c e \mid c t \text { on not } c e
\end{aligned}
$$

The typing environment $H$ is a triple which contains the types of the flow variables, the types of the nodes and the type of the clocks:

$$
\begin{aligned}
H::= & \left(\left[x_{1}: c t_{1}, \ldots, x_{p}: c t_{p}\right],\right. \\
& {\left[f_{1}: \sigma_{1}, \ldots, f_{m}: \sigma_{m}\right], } \\
& {\left.\left[c_{1}: c e_{1}, \ldots, c_{n}: c e_{n}\right]\right) }
\end{aligned}
$$

We use the notation $H+[z: t]$ to add the association $z: t$ to the appropriate part of the triple. We define the notation $[p a t: t]$ as follows:

$[$ pat $: t]= \begin{cases}{[x: t]} & \text { if pat }=x \\ {\left[\text { pat }_{1}: t_{1}\right]+\cdots+\left[\text { pat }_{n}: t_{n}\right]} & \text { if pat }=\left(\text { pat }_{1}, \ldots, \text { pat }_{n}\right) \text { and } t=t_{1} \times \cdots \times t_{n}\end{cases}$

Types can be instantiated and generalized using the following rules:

$$
\begin{aligned}
& \text { inst }\left(\forall \beta_{1}, \ldots, \beta_{m} . \forall \alpha_{1}, \ldots, \alpha_{n} \cdot t\right)= \\
& \quad\left\{t^{\prime} \mid t^{\prime}=t\left[\beta_{1} \leftarrow t_{1}, \ldots, \beta_{m} \leftarrow t_{m}, \alpha_{1} \leftarrow c t_{1}, \ldots, \alpha_{n} \leftarrow c t_{n}\right]\right\} \\
& \text { gen }(t, C)=\forall \beta_{1}, \ldots, \beta_{m} . \forall \alpha_{1}, \ldots, \alpha_{n} \cdot t^{\prime} \\
& \quad \text { where } t^{\prime}=\theta(t) \text { such that } \theta(C) \text { is satisfied } \\
& \quad \text { and }\left\{\beta_{1}, \ldots, \beta_{m}, \alpha_{1}, \ldots, \alpha_{n}\right\}=F V\left(t^{\prime}\right)
\end{aligned}
$$

The typing rules which collect the clocking constraints are given in Figure 7 . The typing rules have the shape $H \vdash e: t \mid C$ which means that in the typing environment $H$, the expression $e$ has type $t$ and must satisfy the set of constraints $C$.

Finally, notice that $c t \times c t \equiv c t$. Therefore, constraints such as $t_{1} \times t_{2}=c t$ can be split into $t_{1}=c t$ and $t_{2}=c t$. 


$$
\begin{aligned}
& H \vdash i: \alpha\left|\emptyset \quad \frac{H \vdash c e}{H \vdash c e: \alpha \mid \emptyset} \quad H \vdash x: H(x)\right| \emptyset \\
& \frac{H \vdash e_{1}: t_{1}\left|C_{1} \quad \cdots \quad H \vdash e_{n}: t_{n}\right| C_{n}}{H \vdash\left(e_{1}, \ldots, e_{n}\right): t_{1} \times \cdots \times t_{n} \mid C_{1} \cup \cdots \cup C_{n}} \\
& \frac{H \vdash e_{1}: t_{1}\left|C_{1} \quad H \vdash e_{2}: t_{2}\right| C_{2}}{H \vdash e_{1} \text { op } e_{2}: c t \mid\left\{t_{1}=t_{2}=c t\right\} \cup C_{1} \cup C_{2}} \\
& H \vdash e: t\left|C \quad H \vdash e_{1}: t_{1}\right| C_{1} \quad H \vdash e_{2}: t_{2} \mid C_{2} \\
& \overline{H \vdash \text { if } e \text { then } e_{1} \text { else } e_{2}: c t \mid\left\{t=t_{1}=t_{2}=c t\right\} \cup C \cup C_{1} \cup C_{2}} \\
& \frac{t_{1} \rightarrow t_{2} \in \operatorname{inst}(H(f)) \quad H \vdash e: t_{3} \mid C}{H \vdash f e: t_{2} \mid\left\{t_{1}=t_{3}\right\} \cup C} \\
& \frac{H \vdash e q s: H^{\prime}\left|C_{1} \quad H+H^{\prime} \vdash e: c t\right| C_{2}}{H \vdash e \text { where rec eqs }: c t \mid C_{1} \cup C_{2}} \\
& \frac{H \vdash e_{1}: t_{1}\left|C_{1} \quad H \vdash e_{2}: t_{2}\right| C_{2}}{H \vdash e_{1} \text { fby } e_{2}: c t \mid\left\{t_{1}=t_{2}=c t\right\} \cup C_{1} \cup C_{2}} \\
& \frac{H \vdash e: t|C \quad H \vdash c e: c t| \emptyset}{H \vdash e \text { when } c e: c t \text { on } c e \mid\{t=c t\} \cup C} \quad \frac{H \vdash e: t|C \quad H \vdash c e: c t| \emptyset}{H \vdash e \text { whenot } c e: c t \text { on not } c e \mid\{t=c t\} \cup C} \\
& H \vdash c e: c t\left|\emptyset \quad H \vdash e_{1}: t_{1}\right| C_{1} \quad H \vdash e_{2}: t_{2} \mid C_{2} \\
& H \vdash \text { merge } c e e_{1} e_{2}: c t \mid\left\{c t \text { on } c e=t_{1}, c t \text { on not } c e=t_{2},\right\} \cup C_{1} \cup C_{2} \\
& \frac{H \vdash e: t \mid C}{H \vdash \operatorname{buffer}(e): \alpha \mid C \cup\{t<: \alpha\}} \\
& \frac{H+[p a t: \beta] \vdash e: t \mid C}{H \vdash p a t=e:[p a t: t] \mid\{\beta=t\} \cup C} \quad \frac{H+H_{2} \vdash e q s_{1}: H_{1}\left|C_{1} \quad H+H_{1} \vdash e q s_{2}: H_{2}\right| C_{2}}{H \vdash e q s_{1} \text { and } e q s_{2}: H_{1}+H_{2} \mid C_{1} \cup C_{2}} \\
& \frac{H+[x: \beta] \vdash e: t \mid C}{H \vdash \text { let node } f(x)=e:[f: \operatorname{gen}(\beta \rightarrow t, C)]} \quad \frac{H \vdash c e}{H \vdash \text { let clock } c=c e:[c: c e]} \\
& \frac{H \vdash d_{1}: H_{1} \quad H+H_{1} \vdash d_{2}: H_{2}}{H \vdash d_{1} ; d_{2}: H_{1}+H_{2}}
\end{aligned}
$$

Fig. 7. Clock type constraints collection. 